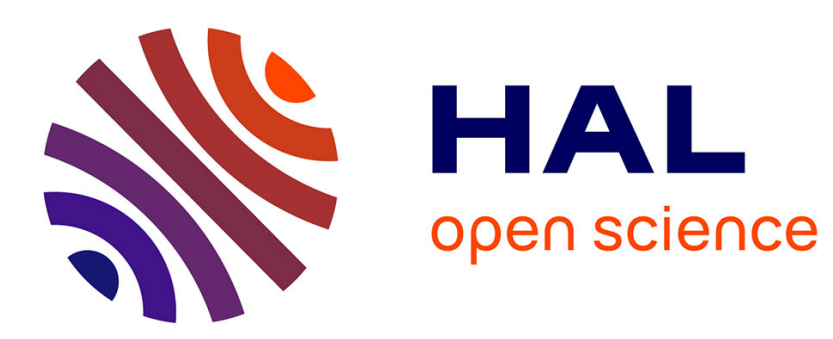

\title{
Coding multitype forests: application to the law of the total population of branching forests
}

\author{
Loïc Chaumont, Rongli Liu
}

\section{To cite this version:}

Loïc Chaumont, Rongli Liu. Coding multitype forests: application to the law of the total population of branching forests. 2013. hal-00783509v2

\section{HAL Id: hal-00783509 \\ https://hal.science/hal-00783509v2}

Preprint submitted on 19 Sep 2013 (v2), last revised 13 Mar 2014 (v3)

HAL is a multi-disciplinary open access archive for the deposit and dissemination of scientific research documents, whether they are published or not. The documents may come from teaching and research institutions in France or abroad, or from public or private research centers.
L'archive ouverte pluridisciplinaire HAL, est destinée au dépôt et à la diffusion de documents scientifiques de niveau recherche, publiés ou non, émanant des établissements d'enseignement et de recherche français ou étrangers, des laboratoires publics ou privés. 


\title{
CODING MULTITYPE FORESTS: APPLICATIONS TO THE LAW OF THE TOTAL PROGENY OF BRANCHING FORESTS AND TO ENUMERATIONS
}

\author{
LOÏC CHAUMONT AND RONGLI LIU
}

\begin{abstract}
By extending the breadth first search algorithm to any $d$-type critical or subcritical irreducible branching forest, we show that such forests may be encoded through $d$ independent, integer valued, $d$-dimensional random walks. An application of this coding together with a multivariate extension of the Ballot Theorem which is proved here, allow us to give an explicit form of the law of the total progeny, jointly with the number of subtrees of each type, in terms of the offspring distribution of the branching process. We then apply these results to some enumeration formulas of multitype forests with given degrees and to a new proof of the Lagrange-Good inversion Theorem.
\end{abstract}

\section{INTRODUCTION}

Let $u_{1}, u_{2} \ldots$ be the labeling in the breadth first search order of the vertices of a critical or subcritical branching forest with progeny distribution $\nu$. Call $p\left(u_{i}\right)$, the size of the progeny of the $i$-th vertex, then the stochastic process $\left(X_{n}\right)_{n \geq 0}$ defined by,

$$
X_{0}=0 \quad \text { and } X_{n+1}-X_{n}=p\left(u_{n+1}\right)-1, \quad n \geq 0
$$

is a downward skip free random walk with step distribution $\mathbb{P}\left(X_{1}=n\right)=\nu(n+1)$, from which the entire structure of the original branching forest can be recovered. We will refer to this random walk as the Lukasiewicz-Harris coding path of the branching forest, see Section 6 of [13], Section 1.1 of [7] or Section 6.2 of [20]. A nice example of application of this coding is that the total progeny of the $k$ first trees $\mathbf{t}_{1}, \mathbf{t}_{2}, \ldots, \mathbf{t}_{k}$ of the forest, see Figure 1, may be expressed as the first passage time of $\left(X_{n}\right)_{n \geq 0}$ at level $-k$, that is,

$$
T_{k}=\inf \left\{i: X_{i}=-k\right\} .
$$

This result combined with the following Kemperman's identity (also known as the Ballot Theorem, see Lemma 5 in [4] or Section 6.2 in [20]):

$$
P\left(T_{k}=n\right)=\frac{k}{n} P\left(X_{n}=-k\right),
$$

allows us to compute the law of the total progeny of $\mathbf{t}_{1}, \mathbf{t}_{2}, \ldots, \mathbf{t}_{k}$ in terms of the progeny distribution $\nu$. Note that the total progeny is actually a functional of the associated branching process, $\left(Z_{n}, n \geq 0\right)$, since the random variable $Z_{n}$ represents the number of individuals at the $n$-th generation in the forest. The expression of this law was first obtained by Otter [19] and Dwass [8].

Date: September 19, 2013.

2010 Mathematics Subject Classification. 60C05, $05 \mathrm{C} 05$.

Key words and phrases. Multitype branching forest, coding, random walks, ballot theorem, total progeny, cyclic exchangeablity.

This work was supported by MODEMAVE research project from the Région Pays de la Loire.

Ce travail a bénécié d'une aide de l'Agence Nationale de la Recherche portant la référence ANR-09BLAN-0084-01. 
Theorem 1.1 (Otter (49) and Dwass (69)). Let $Z=\left(Z_{n}\right)$ be a critical or subcritical branching process. Let $\mathbb{P}_{k}$ be its law when it starts from $Z_{0}=k \geq 1$ and denote by $\nu$ its progeny law. Let $O$ be the total progeny of $Z$, that is $O=\sum_{n \geq 0} Z_{n}$. Then for any $n \geq k$,

$$
\mathbb{P}_{k}(O=n)=\frac{k}{n} \nu^{* n}(n-k),
$$

where $\nu^{* n}$ is the $n$-th iteration of the convolution product of the probability $\nu$ by itself.

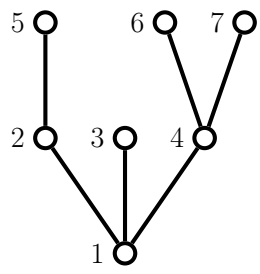

$\mathbf{t}_{1}$
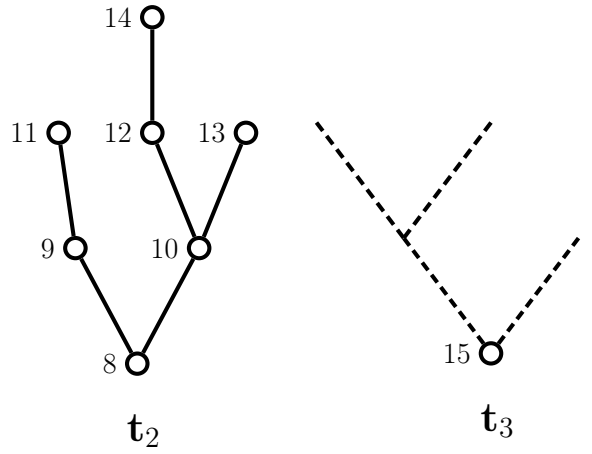

$\mathbf{t}_{3}$

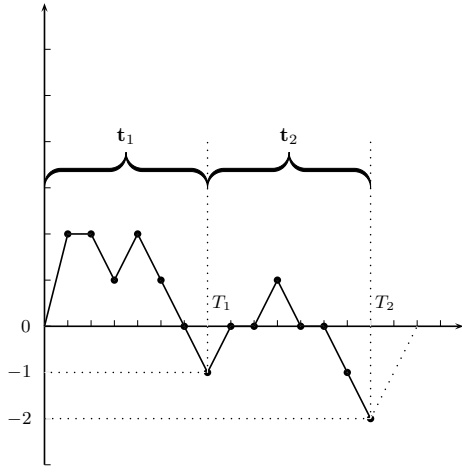

FiguRE 1. A forest labeled according to the breath first search order and the associated Lukasiewicz-Harris coding path.

More generally, whenever a functional of the branching forest admits a 'nice' expression in terms of the Lukasiewicz-Harris coding path, we may expect to obtain an explicit form of its law. For instance, the law of the number of individuals with a given degree in the $k$ first trees can be obtained in this way. We refer to Proposition 1.6 in [14] where the law of the number of leaves, first obtained in [16], is derived from the Lukasiewicz-Harris coding.

As observed in [21], Otter-Dwass and Kemperman's formulas are probabilistic expressions of the Lagrange inversion formula saying that if $g$ is analytic in a neighbourhood of 0 , with $g(0) \neq 0$, then the equation $h(z)=z g(h(z))$ has a unique analytic solution in a neighbourhood of 0 such that

$$
\left[z^{n}\right] h(z)^{k}=\frac{k}{n}\left[z^{n-k}\right] g(z)^{n}
$$

where $\left[z^{i}\right] f(z)$ is the coefficient of $z^{i}$ in the series expansion of $f(z)$. This identity is indeed easily derived from Theorem 1.1 for generating functions of probability distributions with finite support. The general result is then obtained by polynomial continuation.

Then it is well known since a famous paper by Cayley [6] that the Lagrange inversion formula is the analytic counterpart to various enumerations of forests. The link between these enumerations and the Lagrange inversion formula is done through some LukasiewiczHarris type coding paths of forests. We refer to [21], [22], Chapter 6 in [20] and the references therein, for an account on the subject. As an example, we may give the number of labeled forests by degree sequence. Let $N\left(c_{1}, \ldots, c_{n}\right)$ be the number of forests with vertices in the set $\{1, \ldots, n\}$ such that vertex $i \in\{1, \ldots, n\}$ has $c_{i}$ children. The number of trees in this forest is clearly $k:=n-\sum_{i=1}^{n} c_{i}$. Then the following formula will be 
extended in Proposition 5.3 to the multitype case,

$$
N\left(c_{1}, \ldots, c_{n}\right)=\frac{k}{n}\left(\begin{array}{c}
n \\
k
\end{array}\right)\left(\begin{array}{c}
n-k \\
c_{1}, \ldots, c_{n}
\end{array}\right),
$$

where $\left(\begin{array}{c}n-k \\ c_{1}, \ldots, c_{n}\end{array}\right)=\frac{(n-k) !}{c_{1} ! \ldots c_{n} !}$ is the usual multinomial coefficient. A complete account on enumerations of single type forests will be found in [21].

The goal of this paper is to extend the program which is mentioned above to the multitype case. The Lukasiewicz-Harris coding will first be extended to multitype forests and will lead to the bijection stated in Theorem 2.7 between forests and some set of coding sequences. Then in order to obtain the multitype Otter-Dwass identity which is stated in Theorem 1.2, we first need the equivalent of the Ballot Theorem, stated in Theorem 3.4. This theorem together with its equivalent deterministic form, the multivariate Cyclic Lemma 3.3, are actually amongst the most important results of this paper. Then, as a consequence, we will recover some recent results about enumeration of multitype forests and we will present a new proof of the multivariate Lagrange-Good inversion formula. In this section we only mention the extension of Otter-Dwass formula. Ballot Theorem, Cyclic Lemma, enumeration of forests and multivariate Lagrange-Good inversion formula requiring more preliminary results will be stated further in the text.

Let us first set some definitions and notation in multitype branching processes. We set $\mathbb{Z}_{+}=\{0,1,2, \ldots\}$ and $\mathbb{N}=\{1,2, \ldots\}$, and for any integer $n \geq 1$, the set $\{1, \ldots, n\}$ will be denoted by $[n]$. In all the sequel of this paper, $d$ will be an integer such that $d \geq 2$. On a probability space $(\Omega, \mathcal{G}, P)$, we define a $d$-type branching process $\mathbf{Z}:=$ $\left\{\left(Z_{n}^{(1)}, \ldots, Z_{n}^{(d)}\right), n \geq 0\right\}$, as a $\mathbb{Z}_{+}^{d}$ valued Markov chain with transition probabilities:

$$
P\left(\mathbf{Z}_{n+1}=\left(k_{1}, \ldots, k_{d}\right) \mid \mathbf{Z}_{n}=\left(r_{1}, \ldots, r_{d}\right)\right)=\nu_{1}^{* r_{1}} * \cdots * \nu_{d}^{* r_{d}}\left(k_{1}, \ldots, k_{d}\right),
$$

where $\nu_{i}$ are distributions on $\mathbb{Z}_{+}^{d}$ and $\nu_{i}^{* r}$ is the $r$-th iteration of the convolution product of $\nu_{i}$ by itself, with $\nu_{i}^{* 0}=\delta_{0}$. For $\mathrm{r}=\left(r_{1}, \ldots, r_{d}\right) \in \mathbb{Z}_{+}^{d}$, we will denote by $\mathbb{P}_{\mathrm{r}}$ the probability law $P\left(\cdot \mid \mathbf{Z}_{0}=\mathrm{r}\right)$. The vector $\nu=\left(\nu_{1}, \ldots, \nu_{d}\right)$ will be called the progeny distribution of Z. According to this process, each individual of type $i$ gives birth to a random number of children with law $\nu_{i}$, independently of the other individuals of its generation. The integer valued random variable $Z_{n}^{(i)}$ is the total number of individuals of type $i$, at generation $n$. For $i, j \in[d]$, let us define the rate

$$
m_{i j}=\sum_{\mathbf{z} \in \mathbb{Z}_{+}^{d}} z_{j} \nu_{i}(\mathbf{z})
$$

that corresponds to the mean number of children of type $j$, given by an individual of type $i$ and let

$$
\mathbf{M}:=\left(m_{i j}\right)_{i, j \in[d]}
$$

be the mean matrix of $\mathbf{Z}$. Suppose that the extinction time $T$ is a.s. finite, that is

$$
T=\inf \left\{n: \mathbf{Z}_{n}=0\right\}<\infty, \quad \text { a.s. }
$$


Then let $O_{i}$, be the total number of offspring of type $i$, i.e. the total number of individuals of type $i$ which are born up to time $T$ :

$$
O_{i}=\sum_{n=0}^{T} Z_{n}^{(i)}=\sum_{n \geq 0} Z_{n}^{(i)} .
$$

The vector $\left(O_{1}, \ldots, O_{d}\right)$ will be called the total progeny of the multitype branching process.

Up to now, most of the results on the exact law of the total progeny of multitype branching processes concern non irreducible, 2-type branching processes. Let us now recall them. In the case where $d=2$ and when $m_{12}>0$ and $0<m_{11} \leq 1$ but $m_{22}=m_{21}=0$, it may be derived from Theorem 1 (ii) in [4], that the distribution of the total progeny of $\mathbf{Z}$ is given by

$$
\mathbb{P}_{\left(r_{1}, 0\right)}\left(O_{1}=n_{1}, O_{2}=n_{2}\right)=\frac{r_{1}}{n_{1}} \nu_{1}^{* n_{1}}\left(n_{1}-r_{1}, n_{2}\right), \quad 1 \leq r_{1} \leq n_{1} .
$$

When $m_{12}>0$ and $0<m_{11}, m_{22} \leq 1$ but $m_{21}=0$, after some elementary computation, combining the identities in (1.1) and (1.5), we obtain that for $n_{2} \geq 1$,

$$
\mathbb{P}_{\left(r_{1}, 0\right)}\left(O_{1}=n_{1}, O_{2}=n_{2}\right)=\frac{r_{1}}{n_{1} n_{2}} \sum_{j=0}^{n_{2}} j \nu_{1}^{* n_{1}}\left(n_{1}-r_{1}, j\right) \nu_{2}^{* n_{2}}\left(0, n_{2}-j\right) .
$$

Note that (1.5) and (1.6) concern only the reducible case, when $d=2$ and $T<\infty$, a.s. As far as we know, those are the only situations where the law of the total progeny of multitype branching processes is known explicitly.

Recall that if $\mathbf{M}$ is irreducible, then according to Perron-Frobenius Theorem, it admits a unique eigenvalue $\rho$ which is simple, positive and with maximal modulus. In this case, we will also say that $\mathbf{Z}$ is irreducible. If moreover, $\mathbf{Z}$ is non-degenerated, that is, if individuals have exactly one offspring with probability different from 1 , then extinction, that is (1.4), holds if and only if $\rho \leq 1$, see [12], [17] and Chapter $\mathrm{V}$ of [1]. If $\rho=1$, we say that $\mathbf{Z}$ is critical and if $\rho<1$, we say that $\mathbf{Z}$ is subcritical. The results of this paper will be concerned by the case where $\mathbf{Z}$ is irreducible, non-degenerated, and critical or subcritical so that (1.4) holds, that is the multitype branching process $\mathbf{Z}$ becomes extinct with probability 1.

The next result gives the joint law of the total progeny together with the total number of individuals of type $j$, whose parent is of type $i, i \neq j$, up to time $T$. Let us denote by $A_{i j}$ this random variable. We emphasize that the latter $A_{i j}$ is not a functional of the multitype branching process $\mathbf{Z}$. So, its formal definition and the computation of their law require a more complete information provided by the forest. Then Theorem 1.1 and identity (1.6) are extended as follows: 
Theorem 1.2. Assume that the d-type branching process $\mathbf{Z}$ is irreducible, non-degenerated and critical or subcritical. For $i, j \in[d]$, let $O_{i}$ be the total offspring of type $i$, up to the extinction time $T$ and for $i \neq j$, let $A_{i j}$ be the total number of individuals of type $j$, whose parent is of type $i$, up to time $T$.

Then for all integers $r_{i}, n_{i}, k_{i j}, i, j \in[d]$, such that $r_{i} \geq 0, r_{1}+\cdots+r_{d} \geq 1, k_{i j} \geq 0$, for $i \neq j,-k_{j j}=r_{j}+\sum_{i \neq j} k_{i j}$, and $n_{i} \geq-k_{i i}$,

$$
\begin{aligned}
& \mathbb{P}_{\mathrm{r}}\left(O_{1}=n_{1}, \ldots, O_{d}=n_{d}, A_{i j}=k_{i j}, i, j \in[d], i \neq j\right) \\
= & \frac{\operatorname{det}(K)}{\bar{n}_{1} \bar{n}_{2} \ldots \bar{n}_{d}} \prod_{i=1}^{d} \nu_{i}^{* n_{i}}\left(k_{i 1}, \ldots, k_{i(i-1)}, n_{i}+k_{i i}, k_{i(i+1)}, \ldots, k_{i d}\right),
\end{aligned}
$$

where $\mathrm{r}=\left(r_{1}, \ldots, r_{d}\right), \nu_{i}^{* 0}=\delta_{0}, \bar{n}_{i}=n_{i} \vee 1$ and $K$ is the matrix $\left(-k_{i j}\right)_{i, j \in[d]}$ to which we removed the line $i$ and the column $i$, for all $i$ such that $n_{i}=0$.

Our proof of Theorem 1.2 uses a bijection, displayed in Theorem 2.7, between multitype forests and a particular set of multidimensional, integer valued sequences. A consequence of this result is that any critical or subcritical irreducible multitype branching forest is encoded by $d$ independent, $d$-dimensional random walks, see Theorem 3.1. Then, in a similar way to the single type case, the total progeny, jointly with the number of subtrees of each type in the forest, is expressed as the first passage time of this multivariate process in some domain. An extension of the Ballot Theorem, see Theorem 3.4, allows us to conclude as in the single type case. Then we will show in Subsection 5.2 that the multitype Lagrange inversion formula of the generating function of the random vector $\left(O_{1}, \ldots, O_{d}\right)$, obtained by Good [10] is a consequence of Theorem 1.2. Let us also emphasize that our proof of Theorem 1.2 can be adapted in order to deal with the supercritical case and also to drop the assumption of irreducibility. It is only for the sake of simplifying the notation that we have chosen to restrict ourselves to the irreducible critical or subcritical case.

This paper is organized as follows. Section 2 is devoted to deterministic multitype forests. In Subsection 2.1, we present the space of these forests and in Subsection 2.2, we define the space of the coding sequences and we obtain the bijection between this space and the space of multitype forests. This result is stated in Theorem 2.7. Then in Section 3, we define the probability space of multitype branching forests, we display their multitype Lukasiewicz-Harris coding in Theorem 3.1 and we prove its application to the total progeny that is stated in Theorem 1.2. This result requires a multivariate extension of the Ballot Theorem, see Theorem 3.4, whose proof bears on the crucial combinatorial Lemma 3.3. The latter is proved in Section 4. Finally, in Section 5, we recover some existing combinatorial formulas, as applications of our results. In Subsection 5.1, we obtain two formulas for the number of multitype forests with given degrees, as applications of our coding and the combinatorial cyclic Lemma 3.3. Then in Subsection 5.2, we obtain Lagrange-Good inversion formula as a consequence of Theorem 1.2.

\section{Multitype FOREStS}

2.1. The space of multitype forests. A plane forest, is a directed planar graph with no loops $\mathbf{f} \subset \mathbf{v} \times \mathbf{v}$, with a finite or infinite set of vertices $\mathbf{v}=\mathbf{v}(\mathbf{f})$, such that the outer degree of each vertex is equal to 0 or 1 and whose connected components, which are called the trees, are finite. A forest consisting of a single connected component is also called a tree. In a tree $\mathbf{t}$, the only vertex with outer degree equal to 0 is called the root of $\mathbf{t}$. It will be denoted by $r(\mathbf{t})$. The roots of the connected components of a forest $\mathbf{f}$ are called 
the roots of $\mathbf{f}$. For two vertices $u$ and $v$ of a forest $\mathbf{f}$, if $(u, v)$ is a directed edge of $\mathbf{f}$, then we say that $u$ is a child of $v$, or that $v$ is the parent of $u$. The set of plane forests will be denoted by $\mathcal{F}$. The elements of $\mathcal{F}$ will simply be called forests.

We will sometimes have to label the forests, which will be done in the following way. We first give an order to the trees of the forest $\mathbf{f}$ and denote them by $\mathbf{t}_{1}(\mathbf{f}), \mathbf{t}_{2}(\mathbf{f}), \ldots, \mathbf{t}_{k}(\mathbf{f}), \ldots$ (we will usually write $\mathbf{t}_{1}, \mathbf{t}_{2}, \ldots, \mathbf{t}_{k}, \ldots$ if no confusion is possible). Then each tree is labeled according to the breadth first search algorithm: we read the tree from its root to its last generation by running along each generation from the left to the right. This definition should be obvious from the example of Figure 1. If a forest $\mathbf{f}$ contains at least $i$ vertices, then the $i$-th vertex of $\mathbf{f}$ is denoted by $u_{i}(\mathbf{f})$. When no confusion is possible, we will simply denote the $i$-th vertex by $u_{i}$.

Recall that $d$ is an integer such that $d \geq 2$. To each forest $\mathbf{f} \in \mathcal{F}$, we associate an application $c_{\mathbf{f}}: \mathbf{v}(\mathbf{f}) \rightarrow[d]$ such that in the labeling defined above, if $u_{i}, u_{i+1}, \ldots, u_{i+j} \in$ $\mathbf{v}(\mathbf{f})$ have the same parent, then $c_{\mathbf{f}}\left(u_{i}\right) \leq c_{\mathbf{f}}\left(u_{i+1}\right) \leq \cdots \leq c_{\mathbf{f}}\left(u_{i+j}\right)$. For $v \in \mathbf{v}(\mathbf{f})$, the integer $c_{\mathbf{f}}(v)$ is called the type (or the color) of $v$. The couple $\left(\mathbf{f}, c_{\mathbf{f}}\right)$ is called a d-type forest. When no confusion is possible, we will simple write $\mathbf{f}$. The set of $d$-type forests will be denoted by $\mathcal{F}_{d}$. We emphasize that although there is an underlying labeling for each forest, $\mathcal{F}$ and $\mathcal{F}_{d}$ are sets of unlabeled forests. A 2-type forest is represented on Figure 2 below.

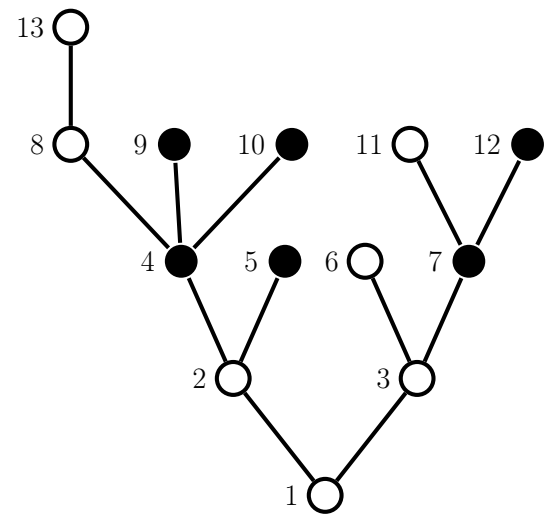

$\mathbf{t}_{1}$
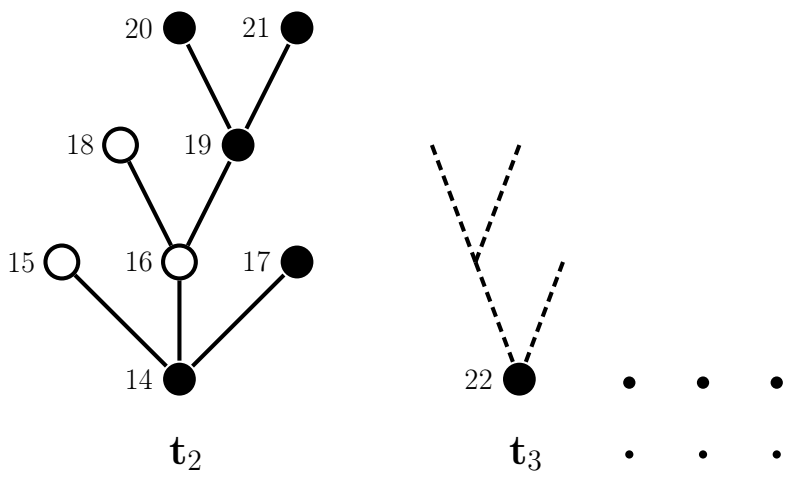

FiguRE 2. A two type forest labeled according to the breath first search order. Vertices of type 1 (resp. 2) are represented in white (resp. black).

A subtree of type $i \in[d]$ of a $d$-type forest $\left(\mathbf{f}, c_{\mathbf{f}}\right) \in \mathcal{F}_{d}$ is a maximal connected subgraph of $\left(\mathbf{f}, c_{\mathbf{f}}\right)$ whose all vertices are of type $i$. Formally, $\mathbf{t}$ is a subtree of type $i$ of $\left(\mathbf{f}, c_{\mathbf{f}}\right)$, if it is a connected subgraph whose all vertices are of type $i$ and such that either $r(\mathbf{t})$ has no parent or the type of its parent is different from $i$. Moreover, if the parent of a vertex $v \in \mathbf{v}(\mathbf{t})^{c}$ belongs to $\mathbf{v}(\mathbf{t})$, then $c_{\mathbf{f}}(v) \neq i$. Subtrees of type $i$ of $\left(\mathbf{f}, c_{\mathbf{f}}\right)$ are ranked according to the order of their roots in $\mathbf{f}$ and are denoted by $\mathbf{t}_{1}^{(i)}, \mathbf{t}_{2}^{(i)}, \ldots, \mathbf{t}_{k}^{(i)}, \ldots$ The forest $\mathbf{f}^{(i)}:=\left\{\mathbf{t}_{1}^{(i)}, \mathbf{t}_{2}^{(i)}, \ldots, \mathbf{t}_{k}^{(i)}, \ldots\right\}$ is called the subforest of type $i$ of $\left(\mathbf{f}, c_{\mathbf{f}}\right)$. It may be considered as an element of $\mathcal{F}$. We denote by $u_{1}^{(i)}, u_{2}^{(i)}, \ldots$ the elements of $\mathbf{v}\left(\mathbf{f}^{(i)}\right)$, ranked in the breadth first search order of $\mathbf{f}^{(i)}$. The subforests of type 1 and 2 of a 2-type forest 
are represented in Figure 3.

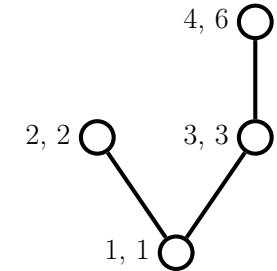

$\mathbf{t}_{1}^{(1)}$

2,9

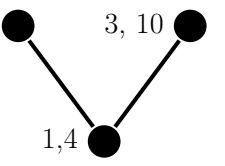

$\mathbf{t}_{1}^{(2)}$

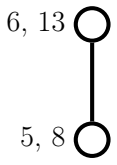

$\mathbf{t}_{2}^{(1)}$

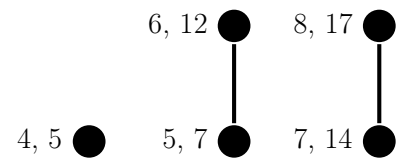

$\mathbf{t}_{2}^{(2)}$
$8,15 \bigcirc$

$\mathbf{t}_{3}^{(1)} \quad \mathbf{t}_{4}^{(1)}$<smiles>[18O]O[18O]</smiles>

$\mathbf{t}_{5}^{(1)} \quad \cdot \quad \cdot$

Figure 3. Labeled subforests $\mathbf{f}^{(1)}$ and $\mathbf{f}^{(2)}$ associated with Figure 2. Beside each vertex, the first number corresponds to its rank in $\mathbf{f}^{(i)}, i=1,2$ and the second one is its rank in the original forest $\mathbf{f}$.

To any forest $\left(\mathbf{f}, c_{\mathbf{f}}\right) \in \mathcal{F}_{d}$, we associate the reduced forest, denoted by $\left(\mathbf{f}_{\mathbf{r}}, c_{\mathbf{f}_{\mathbf{r}}}\right) \in \mathcal{F}_{d}$, which is the forest of $\mathcal{F}_{d}$ obtained by aggregating all the vertices of each subtree of $\left(\mathbf{f}, c_{\mathbf{f}}\right)$ with a given type, in a single vertex with the same type, and preserving an edge between each pair of connected subtrees. An example is given in Figure 4.

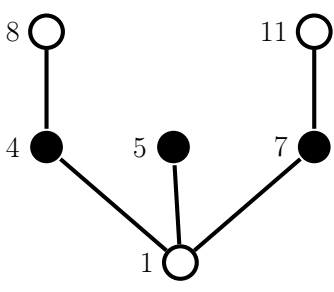

$\mathbf{t}_{1}$

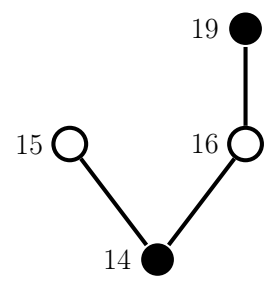

$\mathbf{t}_{2}$

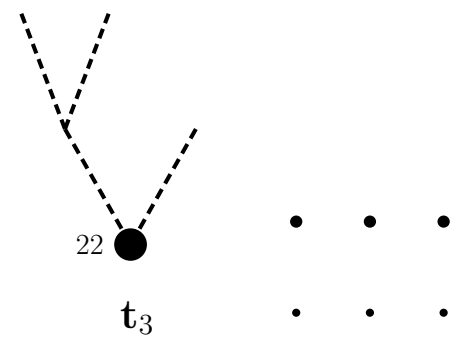

FiguRE 4. Reduced forest associated with the example of Figure 2. Beside each vertex is the rank of the root of the corresponding subtree in the original forest $\mathbf{f}$.

2.2. Coding multitype forests. For a forest $\left(\mathbf{f}, c_{\mathbf{f}}\right) \in \mathcal{F}_{d}$ and $u \in \mathbf{v}(\mathbf{f})$, when no confusion is possible, we denote by $p_{i}(u)$ the number of children of type $i$ of $u$. For each $i \in[d]$, let $n_{i} \geq 0$ be the number of vertices in the subforest $\mathbf{f}^{(i)}$ of $\left(\mathbf{f}, c_{\mathbf{f}}\right)$. Then let us define the $d$-dimensional chain $x^{(i)}=\left(x^{i, 1}, \ldots, x^{i, d}\right)$, with length $n_{i}$ and whose values belong to the set $\mathbb{Z}^{d}$, by $x_{0}^{(i)}=0$ and if $n_{i} \geq 1$,

$$
x_{n+1}^{i, j}-x_{n}^{i, j}=p_{j}\left(u_{n+1}^{(i)}\right), \text { if } i \neq j \text { and } x_{n+1}^{i, i}-x_{n}^{i, i}=p_{i}\left(u_{n+1}^{(i)}\right)-1, \quad 0 \leq n \leq n_{i}-1,
$$


where we recall that $\left(u_{n}^{(i)}\right)_{n \geq 1}$ is the labeling of the subforest $\mathbf{f}^{(i)}$ in its own breadth first search order. Note that the chains $\left(x_{n}^{i, j}\right)$, for $i \neq j$ are nondecreasing whereas $\left(x_{n}^{i, i}\right)$ is a downward skip free chain, i.e. $x_{n+1}^{i, i}-x_{n}^{i, i} \geq-1$, for $0 \leq n \leq n_{i}-1$. The chain $\left(x_{n}^{i, i}\right)$ corresponds to the Lukasiewicz-Harris coding walk of the subforest $\mathbf{f}^{(i)}$, as defined in the introduction, see also Section 6.2 in [20] for a proper definition. In particular, if $n_{i}$ is finite, then $n_{i}=\min \left\{n: x_{n}^{i, i}=\min _{0 \leq k \leq n_{i}} x_{k}^{i, i}\right\}$. These properties of the chains $x^{(i)}$ lead us to the following definition.

Definition 2.1. Let $S_{d}$ be the set of $\left[\mathbb{Z}^{d}\right]^{d}$-valued sequences, $x=\left(x^{(1)}, x^{(2)}, \ldots, x^{(d)}\right)$, such that for all $i \in[d], x^{(i)}=\left(x^{i, 1}, \ldots, x^{i, d}\right)$, is a $\mathbb{Z}^{d}$-valued sequence defined on some interval of integers, $\left\{0,1,2, \ldots, n_{i}\right\}, 0 \leq n_{i} \leq \infty$, which satisfies $x_{0}^{(i)}=0$ and if $n_{i} \geq 1$ then

(i) for $i \neq j$, the sequence $\left(x_{n}^{i, j}\right)_{0 \leq n \leq n_{i}}$ is nondecreasing,

(ii) for all $i, x_{n+1}^{i, i}-x_{n}^{i, i} \geq-1,0 \leq n \leq n_{i}-1$.

We will also denote sequences in $S_{d}$ by $\left(x_{k}^{i, j}, 0 \leq k \leq n_{i}, i, j \in[d]\right)$. The vector $\mathrm{n}=$ $\left(n_{1}, \ldots, n_{d}\right) \in \overline{\mathbb{Z}}_{+}^{d}$, where $\overline{\mathbb{Z}}_{+}=\mathbb{Z}_{+} \cup\{+\infty\}$ will be called the length of $x$.

Relation (2.7) defines an application from the set $\mathcal{F}_{d}$ to the set $S_{d}$. Let us denote by $\Psi$ this application, that is

$$
\begin{aligned}
\Psi: \mathcal{F}_{d} & \rightarrow S_{d} \\
\left(\mathbf{f}, c_{\mathbf{f}}\right) & \mapsto \Psi\left(\left(\mathbf{f}, c_{\mathbf{f}}\right)\right)=x .
\end{aligned}
$$

For $x \in S_{d}$, set $k_{i}=-\inf _{0 \leq n \leq n_{i}} x_{n}^{i, i}$ and define the first passage time process of the chain $\left(x_{n}^{i, i}\right)$ as follows:

$$
\tau_{k}^{(i)}=\min \left\{n \geq 0: x_{n}^{i, i}=-k\right\}, \quad 0 \leq k \leq k_{i},
$$

where $\tau_{k_{i}}^{(i)}=\infty$, if $k_{i}=\infty$. If $x$ is the image by $\Psi$ of a forest $\left(\mathbf{f}, c_{\mathbf{f}}\right) \in \mathcal{F}_{d}$, i.e. $x=\Psi\left(\left(\mathbf{f}, c_{\mathbf{f}}\right)\right)$, then $k_{i}$ is the (finite or infinite) number of trees in the subforest $\mathbf{f}^{(i)}$ and for $k<\infty$, the time $\tau_{k}^{(i)}$ is the total number of vertices which are contained in the $k$ first trees of $\mathbf{f}^{(i)}$, i.e. $\mathbf{t}_{1}^{(i)}, \mathbf{t}_{2}^{(i)}, \ldots, \mathbf{t}_{k}^{(i)}$. This fact is well known and easily follows from the Lukasiewicz-Harris coding of the single type forest $\mathbf{f}^{(i)}$, see the introduction and Lemma 6.3 in [20]. Then for $i, j \in[d]$, define the integer valued sequence

$$
\bar{x}_{k}^{i, j}=x^{i, j}\left(\tau_{k}^{(i)}\right), \quad 0 \leq k \leq k_{i} .
$$

If $x=\Psi\left(\left(\mathbf{f}, c_{\mathbf{f}}\right)\right)$, then we may check that when $i \neq j, \bar{x}_{k}^{i, j}$ is the number of subtrees of type $j$ whose root is the child of a vertex in $\mathbf{t}_{1}^{(i)}, \mathbf{t}_{2}^{(i)}, \ldots, \mathbf{t}_{k}^{(i)}$. Or equivalently, it is the number of vertices of type $j$ whose parent is a vertex of $\mathbf{t}_{1}^{(i)}, \mathbf{t}_{2}^{(i)}, \ldots, \mathbf{t}_{k}^{(i)}$. For each $i \in[d]$, we set

$$
\bar{x}^{(i)}=\left(\bar{x}^{i, 1}, \ldots, \bar{x}^{i, d}\right) \text { and } \bar{x}=\left(\bar{x}^{(1)}, \bar{x}^{(2)}, \ldots, \bar{x}^{(d)}\right) .
$$

Clearly for $i \neq j$, the sequence $\left(\bar{x}_{k}^{i, j}\right)_{0 \leq k \leq k_{i}}$ is increasing and $\bar{x}_{k}^{i, i}=-k$, for all $i \in[d]$ and $0 \leq k \leq k_{i}$, so that $\bar{x} \in S_{d}$ and recalling the definition of the reduced forest, $\left(\mathbf{f}_{\mathbf{r}}, c_{\mathbf{f}_{\mathbf{r}}}\right)$, see the end of Section 2.1, we may check that:

$$
\Psi\left(\left(\mathbf{f}_{\mathbf{r}}, c_{\mathbf{f}_{\mathbf{r}}}\right)\right)=\bar{x} .
$$

For a forest $\left(\mathbf{f}, c_{\mathbf{f}}\right) \in \mathcal{F}_{d}$ with trees $\mathbf{t}_{1}, \mathbf{t}_{2}, \ldots$, we will denote by $\mathrm{c}_{\left(\mathbf{f}, c_{\mathbf{f}}\right)}$ the sequence of types of the roots of $\mathbf{t}_{1}, \mathbf{t}_{2}, \ldots$, i.e.

$$
\mathrm{c}_{\left(\mathbf{f}, c_{\mathbf{f}}\right)}:=\left(c_{\mathbf{f}}\left(r\left(\mathbf{t}_{1}\right)\right), c_{\mathbf{f}}\left(r\left(\mathbf{t}_{2}\right)\right), \ldots\right) .
$$


Note that $\mathrm{c}_{\left(\mathbf{f}, c_{\mathbf{f}}\right)} \in \cup_{1 \leq r \leq \infty}[d]^{r}$ and that $\mathrm{c}_{\left(\mathbf{f}, c_{\mathbf{f}}\right)}=\mathrm{c}_{\left(\mathbf{f}_{\mathbf{r}}, c_{\mathbf{f}_{\mathbf{r}}}\right)}$. When no confusion is possible, $\mathrm{c}_{\left(\mathbf{f}, c_{\mathbf{f}}\right)}$ will simply be denoted by $\mathrm{c}=\left(c_{1}, c_{2}, \ldots\right)$ and we will call it the root type sequence of the forest.

Then before we state the general result on the coding of multitype forests in Theorem 2.7 , we first need to show that the sequences $\left(\bar{x}^{i, j}\right)_{i \neq j}$ together with $\mathrm{c}=\left(c_{1}, c_{2}, \ldots\right)$ allow us to encode the reduced forest $\left(\mathbf{f}_{\mathbf{r}}, c_{\mathbf{f}_{\mathbf{r}}}\right)$, i.e. this forest can be reconstructed from $(\bar{x}, \mathrm{c})$. This claim is stated in Lemma 2.5 below. In order to prove it, we first need to describe the set of sequences which encode reduced forests and to state the preliminary Lemma 2.2 regarding these sequences.

Recall that $\overline{\mathbb{Z}}_{+}=\mathbb{Z}_{+} \cup\{+\infty\}$ and let us define the following (non total) order in $\overline{\mathbb{Z}}_{+}^{d}$ : for two elements $\mathrm{q}=\left(q_{1}, \ldots, q_{d}\right)$ and $\mathrm{q}^{\prime}=\left(q_{1}^{\prime}, \ldots, q_{d}^{\prime}\right)$ of $\overline{\mathbb{Z}}_{+}^{d}$ we write $\mathrm{q} \leq \mathrm{q}^{\prime}$ if $q_{i} \leq q_{i}^{\prime}$ for all $i \in[d]$. Moreover we write $\mathrm{q}<\mathrm{q}^{\prime}$ if $\mathrm{q} \leq \mathrm{q}^{\prime}$ and if there is $i \in[d]$ such that $q_{i}<q_{i}^{\prime}$. For an element $x=\left(x_{k}^{i, j}, 0 \leq k \leq q_{i}, i, j \in[d]\right)$ of $S_{d}$ with length $\mathrm{q}=\left(q_{1}, \ldots, q_{d}\right)$, and $\mathrm{r}=\left(r_{1}, \ldots, r_{d}\right) \in \mathbb{Z}_{+}^{d}$, we say that the system of equations (r, $\left.x\right)$ admits a solution if there exists $\mathrm{s}=\left(s_{1}, \ldots, s_{d}\right) \in \mathbb{Z}_{+}^{d}$, such that $\mathrm{s} \leq \mathrm{q}$ and

$$
r_{j}+\sum_{i=1}^{d} x^{i, j}\left(s_{i}\right)=0, \quad j=1,2, \ldots, d .
$$

Lemma 2.2. Let $x \in S_{d}$ and $\mathrm{r}=\left(r_{1}, \ldots, r_{d}\right) \in \mathbb{Z}_{+}^{d}$. Assume that the system (r, $\left.x\right)$ admits a solution, then

( $i)$ there exists a unique solution $\mathrm{n}=\left(n_{1}, \ldots, n_{d}\right)$ of the system $(\mathrm{r}, x)$ such that if $\mathrm{n}^{\prime}$ is any solution of $(\mathrm{r}, x)$, then $\mathrm{n} \leq \mathrm{n}^{\prime}$. Moreover we have $n_{i}=\min \left\{n: x_{n}^{i, i}=\right.$ $\left.\min _{0 \leq k \leq n_{i}} x_{k}^{i, i}\right\}$, for all $i \in[d]$. A solution such as $\mathrm{n}$ will be called the smallest solution of the system $(\mathrm{r}, x)$.

(ii) Let $\mathrm{r}^{\prime} \in \mathbb{Z}_{+}^{d}$ be such that $\mathrm{r}^{\prime} \leq \mathrm{r}$. Then the system $\left(\mathrm{r}^{\prime}, x\right)$ admits a solution. Let us denote its smallest solution by $\mathrm{n}^{\prime}$. Then the system $\left(\mathrm{r}-\mathrm{r}^{\prime}, \tilde{x}\right)$, where $\tilde{x}^{i, j}(k)=$ $x^{i, j}\left(n_{i}^{\prime}+k\right)-x^{i, j}\left(n_{i}^{\prime}\right), 0 \leq k \leq n_{i}-n_{i}^{\prime}$, admits a solution, and its smallest solution is $\mathrm{n}-\mathrm{n}^{\prime}$.

A proof of this lemma is given in Section 4. For $r=\left(r_{1}, \ldots, r_{d}\right) \in \mathbb{Z}_{+}^{d}$, with $r=r_{1}+\cdots+$ $r_{d} \geq 1$, that is $\mathrm{r}>0$, we define,

$$
C_{d}^{\mathrm{r}}=\left\{c \in[d]^{r}: \text { Card }\left\{j \in[r]: c_{j}=i\right\}=r_{i}, i \in[d]\right\} .
$$

We emphasize that the root type sequence of a forest $\left(\mathbf{f}, c_{\mathbf{f}}\right) \in \mathcal{F}_{d}$ with $r=r_{1}+\cdots+r_{d}$ trees amongst which exactly $r_{i}$ trees have a root of type $i$ is an element $c \in C_{d}^{\mathrm{r}}$. Now we define the subsets of forests and reduced forests whose root type sequence is in $C_{d}^{\mathrm{r}}$ and that contain at least one vertex of each type.

Definition 2.3. Let $\mathrm{r}=\left(r_{1}, \ldots, r_{d}\right) \in \mathbb{Z}_{+}^{d}$, such that $\mathrm{r}>0$.

(i) We denote by $\mathcal{F}_{d}^{\mathrm{r}}$, the subset of $\mathcal{F}_{d}$ of forests $\left(\mathbf{f}, c_{\mathbf{f}}\right)$ with $r_{1}+\cdots+r_{d}$ trees, which contain at least one vertex of each type, and such that $\mathrm{c}_{\left(\mathbf{f}, c_{\mathbf{f}}\right)} \in C_{d}^{\mathrm{r}}$.

(ii) We denote by $\overline{\mathcal{F}}_{d}^{\mathrm{r}}$ the subset of $\mathcal{F}_{d}^{\mathrm{r}}$, of reduced forests, more specifically, $\left(\mathbf{f}, c_{\mathbf{f}}\right) \in \overline{\mathcal{F}}_{d}^{\mathrm{r}}$ if $\left(\mathbf{f}, c_{\mathbf{f}}\right) \in \mathcal{F}_{d}^{\mathrm{r}}$ and if for each $i$, vertices of type $i \in[d]$ in $\mathbf{v}(\mathbf{f})$ have no child of type $i$.

Then we define the sets of coding sequences related to $\mathcal{F}_{d}^{\mathrm{r}}$ and $\overline{\mathcal{F}}_{d}^{\mathrm{r}}$. 
Definition 2.4. Let $\mathrm{r}=\left(r_{1}, \ldots, r_{d}\right) \in \mathbb{Z}_{+}^{d}$, such that $\mathrm{r}>0$.

(i) We denote by $S_{d}^{\mathrm{r}}$ the subset of $S_{d}$ of sequences $x$ whose length belongs to $\mathbb{N}^{d}$ and corresponds to the smallest solution of the system ( $\mathrm{r}, x)$ defined in (2.12).

(i) We denote by $\bar{S}_{d}^{\mathrm{r}}$ the subset of $S_{d}^{\mathrm{r}}$ consisting in sequences, such that $x_{k}^{i, i}=-k$, for all $k$ and $i$.

Then we first establish a bijection between the sets $\overline{\mathcal{F}}_{d}^{\mathrm{r}}$ and $\bar{S}_{d}^{\mathrm{r}} \times C_{d}^{\mathrm{r}}$. Recall the definition of $\Psi$ in (2.8).

Lemma 2.5. Let $\mathrm{r}=\left(r_{1}, \ldots, r_{d}\right) \in \mathbb{Z}_{+}^{d}$, be such that $\mathrm{r}>0$, then the mapping

$$
\begin{aligned}
\Phi: \overline{\mathcal{F}}_{d}^{\mathrm{r}} & \rightarrow \bar{S}_{d}^{\mathrm{r}} \times C_{d}^{\mathrm{r}} \\
\left(\mathbf{f}, c_{\mathbf{f}}\right) & \mapsto\left(\Psi\left(\left(\mathbf{f}, c_{\mathbf{f}}\right)\right), \mathrm{c}_{\left(\mathbf{f}, c_{\mathbf{f}}\right)}\right)
\end{aligned}
$$

is a bijection.

Proof. Let $\left(\mathbf{f}, c_{\mathbf{f}}\right) \in \overline{\mathcal{F}}_{d}^{\mathrm{r}}$ and let $k_{i}$ be the total number of subtrees of type $i$ which are contained in $\left(\mathbf{f}, c_{\mathbf{f}}\right)$. (Note that since $\left(\mathbf{f}, c_{\mathbf{f}}\right)$ is a reduced forest, its subtrees are actually single vertices.) By definition, $k_{i} \geq 1$, for each $i$. The fact that, $\mathrm{c}_{\left(\mathbf{f}, c_{\mathbf{f}}\right)} \in C_{d}^{\mathrm{r}}$ follows from Definition $2.3(i i)$. Then let us show that $x=\Psi\left(\left(\mathbf{f}, c_{\mathbf{f}}\right)\right) \in \bar{S}_{d}^{\mathrm{r}}$. Since $\left(\mathbf{f}, c_{\mathbf{f}}\right)$ is a reduced forest, then $x=\bar{x}$. Besides, from $(2.10), x$ has length $\mathrm{k}=\left(k_{1}, \ldots, k_{d}\right)$ and for $j \neq i, x^{i, j}\left(k_{i}\right)$ is the number of subtrees of type $j$ whose root is the child of a vertex of $\left\{\mathbf{t}_{1}^{(i)}, \mathbf{t}_{2}^{(i)}, \ldots, \mathbf{t}_{k_{i}}^{(i)}\right\}$, i.e. of any subtree of type $i$ in $\left(\mathbf{f}, c_{\mathbf{f}}\right)$. Hence for $j \in[d], \sum_{i \neq j} x^{i, j}\left(k_{i}\right)$ is the total number of subtrees of type $j$ in $\left(\mathbf{f}, c_{\mathbf{f}}\right)$, whose root is the child of a vertex of type $i \in[d], i \neq j$. Then we obtain $k_{j}$ by adding to $\sum_{i \neq j} x^{i, j}\left(k_{i}\right)$, the $r_{j}$ subtrees of type $j$ whose root is one of the roots of $\mathbf{t}_{1}, \ldots, \mathbf{t}_{r}$, where $r=r_{1}+\cdots+r_{d}$. That is $k_{j}=r_{j}+\sum_{i \neq j} x^{i, j}\left(k_{i}\right)$. Since moreover from $(2.10), x_{k}^{i, i}=-k$, for all $0 \leq k \leq k_{i}$, we have proved that $\mathrm{k}$ is a solution of the system $(\mathrm{r}, x)$. It remains to prove that it is the smallest solution.

Let us first assume that $\mathrm{r}=(1,0, \ldots, 0)$, so that $\left(\mathbf{f}, c_{\mathbf{f}}\right)$ consists in a single tree $\mathbf{t}_{1}$ whose root has color $c_{\mathbf{f}}\left(r\left(\mathbf{t}_{1}\right)\right)=1$. Then we can reconstruct, this tree from the $d$ sequences $\left(x_{k}^{(i)}, 0 \leq k \leq k_{i}\right), i \in[d]$ by inverting the procedure defined in (2.7) and this reconstruction procedure gives a unique tree. Indeed, by definition of the application $\Psi$, each sequence $\left(x_{k}^{(i)}, 0 \leq k \leq k_{i}\right)$, is associated to a unique 'marked subforest', say $\tilde{\mathbf{f}}^{(i)}$, of type $i$ whose vertices kept the memory of their progeny. More specifically, for $k \in\left[k_{i}\right]$, the increment $x_{k}^{(i)}-x_{k-1}^{(i)}$ gives the progeny of the $k$-th vertex of the subforest $\mathbf{f}^{(i)}$. This connection between marked subforests $\tilde{\mathbf{f}}^{(i)}$ and sequences $\left(x_{k}^{(i)}, 0 \leq k \leq k_{i}\right)$ is illustrated on Figure 5 . Now let $\mathrm{k}^{\prime} \leq \mathrm{k}$ be the smallest solution of the system $(\mathrm{r}, x)$. Let $\mathrm{q}=\left(q_{1}, \ldots, q_{d}\right)<\mathrm{k}^{\prime}$ and suppose that we have been able to perform the reconstruction procedure until q, that is from the sequences $\left(x_{k}^{(i)}, 0 \leq k \leq q_{i}\right), i \in[d]$. Then since $\mathrm{q}$ is not a solution of $(\mathrm{r}, x)$, we see from what has been proved just above that the tree that is obtained is 'not complete'. That is, at least one of its leaves (say of type $j$ ) is marked, so that this leaf should still get children whose types and numbers are given by the next jump $x_{q_{j}+1}^{(j)}-x_{q_{j}}^{(j)}$, for $q_{j}<k_{j}^{\prime}$, according to the reconstruction procedure. Thus, doing so, we necessarily end up with a tree from the sequences $\left(x_{k}^{(i)}, 0 \leq k \leq k_{i}^{\prime}\right), i \in[d]$, and this tree is complete, that is none of its leaves is marked. Then since the reconstruction procedure obtained by inverting (2.7), gives a unique tree, we necessarily have $\mathrm{k}^{\prime}=\mathrm{k}$.

Then let $\mathrm{r}=\left(r_{1}, \ldots, r_{d}\right) \in \mathbb{Z}_{+}^{d}$. Assume with no loss of generality that the root of the first tree $\mathbf{t}_{1}$ of $\left(\mathbf{f}, c_{\mathbf{f}}\right)$ has color 1 . Let $k_{i}^{1}$ be the number of subtrees of type $i$ in $\mathbf{t}_{1}$. 


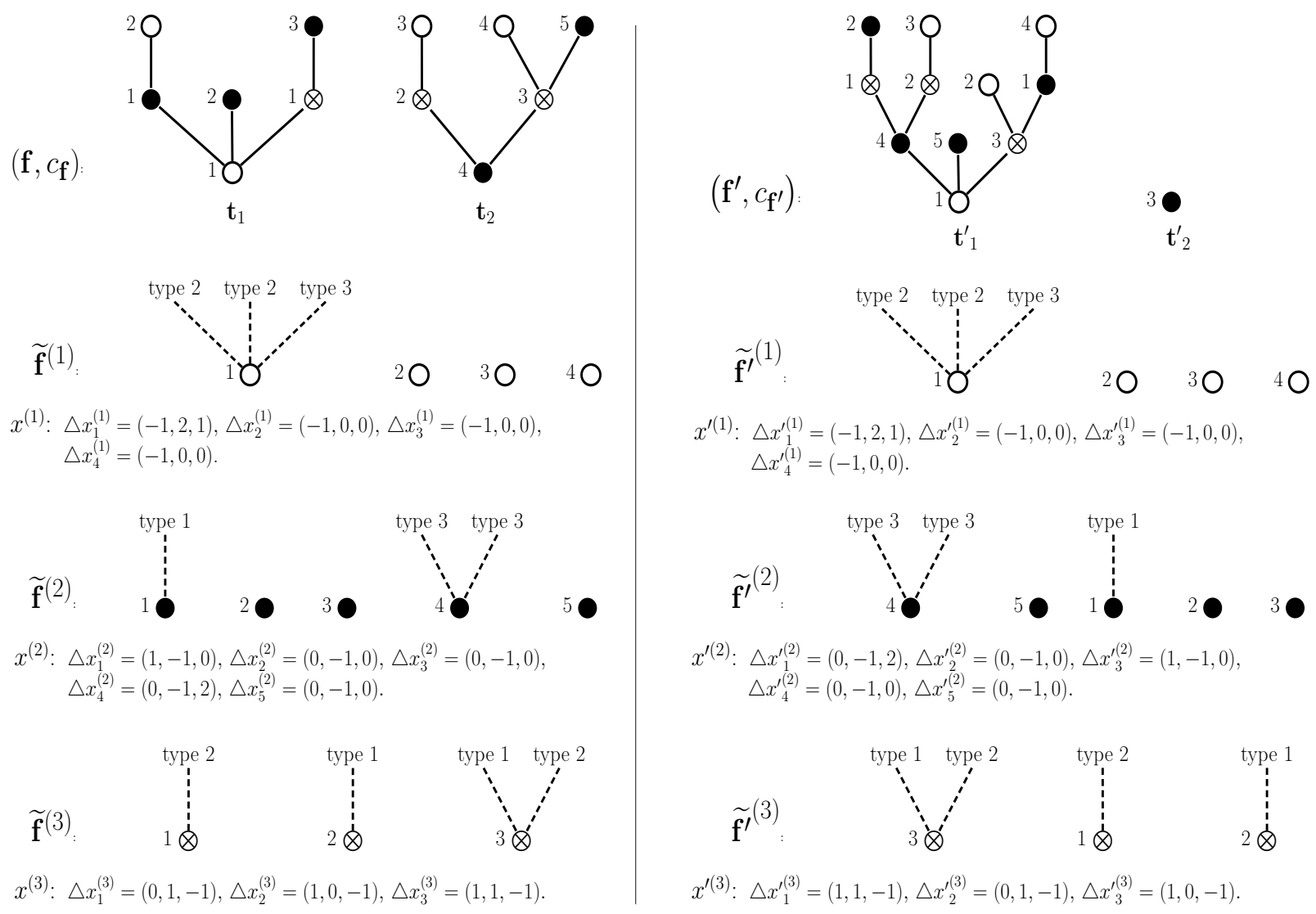

FiguRE 5. On the left, a three types reduced forest $\left(\mathbf{f}, c_{\mathbf{f}}\right)$ (the labelling is related to each subforest, see Subsection 2.1), the three marked subforests $\tilde{\mathbf{f}}^{(1)}, \tilde{\mathbf{f}}^{(2)}, \tilde{\mathbf{f}}^{(3)}$ of $\left(\mathbf{f}, c_{\mathbf{f}}\right)$ and the coding sequences $x^{(1)}, x^{(2)}, x^{(3)}$. Here we have set $\Delta x_{k}^{(i)}=x_{k}^{(i)}-x_{k-1}^{(i)}$. On the right, a three types reduced forest $\left(\mathbf{f}^{\prime}, c_{\mathbf{f}^{\prime}}\right)$ obtained after cyclical permutations of $\tilde{\mathbf{f}}^{(1)}, \tilde{\mathbf{f}}^{(2)}, \tilde{\mathbf{f}}^{(3)}$.

From Lemma 2.2, the system $\left(\mathrm{r}^{1}, x\right)$, where $\mathrm{r}^{1}:=(1,0, \ldots, 0)$, admits a smallest solution. Moreover from the reconstruction procedure which is described above, this solution is $\mathrm{k}^{1}=\left(k_{1}^{1}, \ldots, k_{d}^{1}\right)$. Suppose now with no loss of generality that the second tree, $\mathbf{t}_{2}$ in $\left(\mathbf{f}, c_{\mathbf{f}}\right)$ has color 2 . Let $k_{i}^{2}$ be the number of subtrees of type $i$ in $\mathbf{t}_{2}$. Then from the same arguments as for the reconstruction of the first tree, $\mathbf{t}_{2}$ may be reconstructed from the system $\left(\mathrm{r}^{2}, y\right)$, where $\mathrm{r}^{2}:=(0,1,0, \ldots, 0)$ and $y^{i, j}(k)=x^{i, j}\left(k_{i}^{1}+k\right)-x^{i, j}\left(k_{i}^{1}\right), k \geq 0$. Moreover $\left(\mathrm{r}^{2}, y\right)$ admits $\mathrm{k}^{2}=\left(k_{1}^{2}, \ldots, k_{d}^{2}\right)$ as a smallest solution. Then from part $(i i)$ of Lemma $2.2, \mathrm{k}^{1}+\mathrm{k}^{2}$ is the smallest solution of the system $\left(\mathrm{r}^{1}+\mathrm{r}^{2}, x\right)$. So we have proved the result for the forest consisting in the trees $\mathbf{t}_{1}$ and $\mathbf{t}_{2}$. Then by iterating these arguments for each tree of $\left(\mathbf{f}, c_{\mathbf{f}}\right)$, we obtain that $x \in \bar{S}_{d}^{\mathrm{r}}$.

Conversely, let $\mathrm{c}=\left(c_{1}, \ldots, c_{r}\right) \in C_{d}^{\mathrm{r}}, x \in \bar{S}_{d}^{\mathrm{r}}$ and let $\mathrm{k}=\left(k_{1}, \ldots, k_{d}\right)$ be the smallest solution of the system $(\mathrm{r}, x)$. Then let us show that there is a forest $\left(\mathbf{f}, c_{\mathbf{f}}\right) \in \overline{\mathcal{F}}_{d}^{\mathrm{r}}$ such that $\Psi\left(\left(\mathbf{f}, c_{\mathbf{f}}\right)\right)=x$ and $\mathrm{c}_{\left(\mathbf{f}, c_{\mathbf{f}}\right)}=\mathrm{c}$. Assume, without loss of generality that $c_{1}=1$. From Lemma $2.2(i i)$, there is a smallest solution, say $\mathrm{k}^{1}=\left(k_{1}^{1}, \ldots, k_{d}^{1}\right)$, to the system $\left(\mathrm{r}^{1}, x\right)$, where $\mathrm{r}^{1}:=(1,0, \ldots, 0)$. Then we may reconstruct a unique forest $\left(\mathbf{t}_{1}, c_{\mathbf{t}_{1}}\right) \in \overline{\mathcal{F}}_{d}^{1}$ (consisting in a single tree) such that $\Psi\left(\left(\mathbf{t}_{1}, c_{\mathbf{t}_{1}}\right)\right)=\left(x_{k}^{i, j}, 0 \leq k \leq k_{i}^{1}, i, j \in[d]\right)$ and $\mathrm{c}_{\left(\mathbf{t}_{1}, c_{\mathbf{t}_{1}}\right)}=1$ 
by inverting the procedure that is described in (2.7). Assume for instance that $c_{2}=2$ and set $\mathrm{r}^{2}:=(1,1,0, \ldots, 0) \leq \mathrm{r}$, then from Lemma $2.2(\mathrm{ii})$, there is a smallest solution, say $\mathrm{k}^{2}$, to the system $\left(\mathrm{r}^{2}, x\right)$. Moreover, $\mathrm{k}^{3}=\mathrm{k}^{2}-\mathrm{k}^{1}$ is the smallest solution of the system $\left(\mathrm{r}^{2}-\mathrm{r}^{1}, y\right)$, where $y^{i, j}(k)=x^{i, j}\left(k_{i}^{1}+k\right)-x^{i, j}\left(k_{i}^{1}\right), k \geq 0$. Then as before, we can reconstruct a unique tree $\left(\mathbf{t}_{2}, c_{\mathbf{t}_{2}}\right)$ such that $\Psi\left(\left(\mathbf{t}_{2}, c_{\mathbf{t}_{2}}\right)\right)=y$ and such that the forest $\hat{\mathbf{f}}=\left\{\mathbf{t}_{1}, \mathbf{t}_{2}\right\}$ satisfies $\Psi\left(\left(\hat{\mathbf{f}}, c_{\hat{\mathbf{f}}}\right)\right)=\left(x_{k}^{i, j}, 0 \leq k \leq k_{i}^{2}, i, j \in[d]\right)$ and $\mathrm{c}_{\left(\hat{\mathbf{f}}, c_{\hat{\mathbf{f}}}\right)}=(1,1,0, \ldots, 0)$. Then iterating these arguments, we may reconstruct a unique forest $\left(\mathbf{f}, c_{\mathbf{f}}\right) \in \mathcal{F}_{d}^{\mathrm{r}}$ such that $\Psi\left(\left(\mathbf{f}, c_{\mathbf{f}}\right)\right)=x$ and $\mathrm{c}_{\left(\mathbf{f}, c_{\mathbf{f}}\right)}=c$.

Let $x \in S_{d}$ with length $\mathrm{n}=\left(n_{1}, \ldots, n_{d}\right)$ and recall from $(2.10)$, the definition of the associated sequence $\bar{x}$, with length $\mathrm{k}=\left(k_{1}, \ldots, k_{d}\right)$, such that $k_{i}=-\min _{0 \leq n \leq n_{i}} x_{n}^{i, i}$.

Lemma 2.6. Let $\mathrm{r} \in \mathbb{Z}_{+}^{d}$, such that $\mathrm{r}>0$ and $x \in S_{d}$, with length $\mathrm{n} \in \mathbb{N}^{d}$ and set $k_{i}=-\min _{0 \leq n \leq n_{i}} x_{n}^{i, i}, i \in[d]$. If $\mathrm{n}$ is the smallest solution of the system (r, $x$ ) (i.e. $\left.x \in S_{d}^{\mathrm{r}}\right)$, then $\mathrm{k}=\left(k_{1}, \ldots, k_{d}\right)$ is the smallest solution of the system $(\mathrm{r}, \bar{x})$. Conversely, if $n_{i}=\tau_{k_{i}}^{(i)}$, for all $i \in[d]$ and if $\mathrm{k}$ is the smallest solution of $(\mathrm{r}, \bar{x})$, (i.e. $\left.\bar{x} \in \bar{S}_{d}^{\mathrm{r}}\right)$, then $\mathrm{n}$ is the smallest solution of $(\mathrm{r}, x)$.

Proof. Assume that $\mathrm{n}$ is the smallest solution of the system (r, $x)$. Then from part $(i)$ of Lemma $2.2, n_{i}=\tau_{k_{i}}^{(i)}$, hence $\mathrm{k}$ is a solution of $(\mathrm{r}, \bar{x})$. Let $\mathrm{k}^{\prime} \leq \mathrm{k}$ be such that

$$
r_{j}+\sum_{i=1}^{d} \bar{x}^{i, j}\left(k_{i}^{\prime}\right)=0, \quad j=1,2, \ldots d .
$$

Then by definition of $\bar{x}$ there is $\mathrm{n}^{\prime} \leq \mathrm{n}$ such that $n_{i}^{\prime}=\tau_{k_{i}^{\prime}}^{(i)}$ and

$$
r_{j}+\sum_{i=1}^{d} x^{i, j}\left(n_{i}^{\prime}\right)=0, \quad j=1,2, \ldots d .
$$

So $\mathrm{n}^{\prime}=\mathrm{n}$ and hence $\mathrm{k}^{\prime}=\mathrm{k}$.

The converse is proved in the same way. Suppose that $n_{i}=\tau_{k_{i}}^{(i)}, i \in[d]$, and that $\mathrm{k}$ is the smallest solution of $(\mathrm{r}, \bar{x})$. Then clearly, $\mathrm{n}$ is a solution of $(\mathrm{r}, x)$. Let $\mathrm{n}^{\prime}$ be the smallest solution of $(\mathrm{r}, x)$. Then from Lemma 2.2 , there is $\mathrm{k}^{\prime}$ such that $n_{i}^{\prime}=\tau_{k_{i}^{\prime}}^{(i)}$, hence $\mathrm{k}^{\prime}$ is a solution of $(\mathrm{r}, \bar{x})$. This implies that $\mathrm{k} \leq \mathrm{k}^{\prime}$, so that $\mathrm{n} \leq \mathrm{n}^{\prime}$, hence $\mathrm{n}=\mathrm{n}^{\prime}$.

Now we extend the application $\Phi$ defined in Lemma 2.5 to the set $\mathcal{F}_{d}^{\mathrm{r}}$. Here is the main result of this section, that can be considered as an extension of Proposition 1.1 in [7].

Theorem 2.7. Let $\mathrm{r}=\left(r_{1}, \ldots, r_{d}\right) \in \mathbb{Z}_{+}^{d}$, be such that $\mathrm{r}>0$, then the mapping

$$
\begin{aligned}
\Phi: \mathcal{F}_{d}^{\mathrm{r}} & \rightarrow S_{d}^{\mathrm{r}} \times C_{d}^{\mathrm{r}} \\
\left(\mathbf{f}, c_{\mathbf{f}}\right) & \mapsto\left(\Psi\left(\left(\mathbf{f}, c_{\mathbf{f}}\right)\right), \mathrm{c}_{\left(\mathbf{f}, c_{\mathbf{f}}\right)}\right)
\end{aligned}
$$

is a bijection.

Proof. Let us first check that for any $\left(\mathbf{f}, c_{\mathbf{f}}\right) \in \mathcal{F}_{d}^{\mathrm{r}}$, we have $\Phi\left(\left(\mathbf{f}, c_{\mathbf{f}}\right)\right) \in S_{d}^{\mathrm{r}} \times C_{d}^{\mathrm{r}}$. By definition 2.3, $(i), \mathrm{c}_{\left(\mathbf{f}, c_{\mathbf{f}}\right)} \in C_{d}^{\mathrm{r}}$. Now set $x=\Psi\left(\left(\mathbf{f}, c_{\mathbf{f}}\right)\right)$ and let $\left(\mathbf{f}_{\mathbf{r}}, c_{\mathbf{f}_{\mathbf{r}}}\right) \in \overline{\mathcal{F}}_{d}^{\mathrm{r}}$ be the forest, $\left(\mathbf{f}, c_{\mathbf{f}}\right)$ once reduced. Then from (2.11) and Lemma 2.5, this reduced forest is encoded by $\left(\bar{x}, c_{\mathbf{f}}\right)$. Let $\mathrm{k}=\left(k_{1}, \ldots, k_{d}\right)$ be the number of subtrees of type $i$ in this forest (this is actually the number of vertices of type $i$ ), then $\mathrm{k}$ is the length of $\bar{x}$ and it is the smallest solution of $(\mathrm{r}, \bar{x})$, i.e. $\bar{x} \in \bar{S}_{d}^{\mathrm{r}}$. Moreover $\mathrm{n}=\left(n_{1}, \ldots, n_{d}\right)$, where $n_{i}=\tau_{k_{i}}^{(i)}$ is the length 
of $x$, and from Lemma 2.6, it is the smallest solution of $(\mathrm{r}, x)$. So, we have proved that $\Psi\left(\left(\mathbf{f}, c_{\mathbf{f}}\right)\right) \in S_{d}^{\mathrm{r}}$.

Conversely let $(x, \mathrm{c}) \in S_{d}^{\mathrm{r}} \times C_{d}^{\mathrm{r}}$. From Lemma 2.5 , to $(\bar{x}, \mathrm{c})$, we may associate a unique forest $\left(\mathbf{f}_{\mathbf{r}}, c_{\mathbf{f}_{\mathbf{r}}}\right) \in \overline{\mathcal{F}}_{d}^{\mathrm{r}}$. Then let $k_{i}$ be the number of vertices of type $i$ in this forest. For $k \in\left[k_{i}\right]$, let $u_{k}^{(i)}$ be the $k$-th vertex of type $i$ in the breadth first search order of $\left(\mathbf{f}_{\mathbf{r}}, c_{\mathbf{f}_{\mathbf{r}}}\right)$. Then in $\left(\mathbf{f}_{\mathbf{r}}, c_{\mathbf{f}_{\mathbf{r}}}\right)$, we replace the vertex $u_{k}^{(i)}$ by the subtree of type $i$ which is encoded by the Lukasiewicz-Harris path $\left(x^{i, i}\left(\tau_{k-1}^{(i)}+l\right)+k-1,0 \leq l \leq \tau_{k}^{(i)}-\tau_{k-1}^{(i)}\right)$. We know about the progeny of each vertex of this subtree, thanks to the chains $\left(x^{i, j}\left(\tau_{k-1}^{(i)}+l\right)-x^{i, j}\left(\tau_{k-1}^{(i)}\right), 0 \leq\right.$ $\left.l \leq \tau_{k}^{(i)}-\tau_{k-1}^{(i)}\right)$, so that we can graft at the proper place, on this subtree, all the corresponding subtrees of the other types which have been constructed from the same procedure. Proceeding this way, we construct a unique forest $\left(\mathbf{f}, c_{\mathbf{f}}\right) \in \mathcal{F}_{d}^{\mathrm{r}}$ and we easily check that $\Psi\left(\left(\mathbf{f}, c_{\mathbf{f}}\right)\right)=x$.

\section{Multitype BRAnChing trees And FOREStS}

Let $\nu_{i}, i \in[d]$ be distributions on $\mathbb{Z}_{+}^{d}$, such that $\nu=\left(\nu_{1}, \ldots, \nu_{d}\right)$ is the progeny law of an irreducible, critical or subcritical, non-degenerated branching process, as defined in Section 1. Assume that we can define on the reference probability space $(\Omega, \mathcal{G}, P)$ intro-

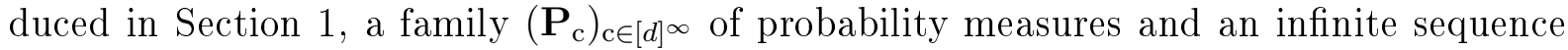
$\mathrm{F}=\left(\mathrm{T}_{k}\right)_{k \geq 1}$ of independent random trees, such that for each $\mathrm{c}=\left(c_{1}, c_{2}, \ldots\right) \in[d]^{\infty}$ and $k \geq 1$, under $\mathbf{P}_{\mathrm{c}}, \mathrm{T}_{k}$ is a branching tree, with progeny law $\nu$, whose root has type $r\left(\mathrm{~T}_{k}\right)=c_{k}$. In particular, for any random time $\alpha:(\Omega, \mathcal{G}) \rightarrow \mathbb{N} \cup\{+\infty\}$, the sequence $\left\{\mathrm{T}_{1}, \ldots, \mathrm{T}_{\alpha}\right\}$ is an element of $\mathcal{F}_{d}$. The infinite sequence $\mathrm{F}$ will be called a $d$-type branching forest with progeny law $\nu$.

Let us denote by $\mathrm{F}^{(i)}$ the subforest of type $i$ of $\mathrm{F}$, as it is defined in subsection 2.1. From the properties of $\nu$, it follows that for each $i \in[d]$, the subforest $\mathrm{F}^{(i)}$ is a.s. infinite, so that we may define a $\mathbb{Z}^{d}$ valued infinite random sequence $X^{(i)}=\left(X^{i, 1}, \ldots, X^{i, d}\right)$, for $i \in[d]$, in the same way as in $(2.7)$, that is $X_{0}^{(i)}=0$ and

$$
X_{n+1}^{i, j}-X_{n}^{i, j}=p_{j}\left(\mathrm{u}_{n+1}^{(i)}\right), \quad \text { if } i \neq j \quad \text { and } \quad X_{n+1}^{i, i}-X_{n}^{i, i}=p_{i}\left(\mathrm{u}_{n+1}^{(i)}\right)-1, \quad n \geq 0,
$$

where $\left(\mathrm{u}_{n}^{(i)}\right)_{n \geq 1}$ is the labeling of $\mathrm{F}^{(i)}$ in its breadth first search order.

Theorem 3.1. Let $\mathrm{F}$ be a d-type branching forest with progeny law $\nu$.

1. Then for any $\mathrm{c}=\left(c_{1}, c_{2} \ldots\right) \in[d]^{\infty}$, under $\mathbf{P}_{\mathrm{c}}$, the chains

$$
X^{(i)}=\left(X^{i, 1}, \ldots, X^{i, d}\right), \quad i=1, \ldots, d
$$

are independent random walks with step distribution

$$
\mathbf{P}_{\mathrm{c}}\left(X_{1}^{(i)}=\left(q_{1}, \ldots, q_{d}\right)\right)=\nu_{i}\left(q_{1}, \ldots, q_{i-1}, q_{i}+1, q_{i+1}, \ldots, q_{d}\right) .
$$

In particular, their laws do not depend on c. For each $i \in[d], X^{i, i}$ is a downward skip free random walk such that $\liminf _{n \rightarrow+\infty} X_{n}^{i, i}=-\infty$, a.s. and for $j \neq i, X^{i, j}$ is a renewal process.

2. For all integer $r \geq 1$, almost surely there is $\mathrm{r} \in \mathbb{Z}_{+}^{d}$, with $r=r_{1}+\cdots+r_{d}$ and such that there is a smallest solution $\mathrm{n}$ to the system $(\mathrm{r}, X)$.

3. Conversely, let $Y$ be a copy of $X$ and $\mathrm{c}=\left(c_{1}, c_{2} \ldots\right) \in[d]^{\infty}$. Then to $Y$ and $\mathrm{c}$, we may associate a unique d-type branching forest, with progeny law $\nu$ and root type sequence c, whose coding random walk is $Y$. 
Proof. Part 1. just follows from the construction (3.13) of $X$. Since the order on the subforests $\mathrm{F}^{(i)}$ does not depend on the particular topology of $\mathrm{F}$, from the branching property, it is clear that the chains $X^{(i)}, i \in[d]$ are independent random walks. Then the expression of the law of $X^{(i)}$ is a direct consequence of (3.13). Recall that $X^{i, i}$ is the Lukasiewicz-Harris path of the subforest $\mathrm{F}^{(i)}$, see section 6.2 of [20]. Moreover, since from the properties of $\nu$, each subforest $\mathrm{F}^{(i)}$ is a.s. infinite, the random walk $X^{i, i}$ satisfies $\lim \inf _{n \rightarrow \infty} X_{n}^{i, i}=-\infty$, a.s. The fact that $X^{i, j}$, for $i \neq j$ is a renewal process is obvious.

Then part 2 is a direct consequence of the construction of $X$ and Theorem 2.7. Let $r \geq 1$ and first assume that the finite forest $\left\{\mathrm{T}_{1}, \ldots, \mathrm{T}_{r}\right\}$ consisting in the $r$ first trees of $\mathrm{F}$ contains at least one vertex of each type. Let $\mathrm{r}$ be the unique element of $\mathbb{Z}^{d}$ such that $\left\{\mathrm{T}_{1}, \ldots, \mathrm{T}_{r}\right\} \in \mathcal{F}_{d}^{\mathrm{r}}$. Then, by coding the forest $\left\{\mathrm{T}_{1}, \ldots, \mathrm{T}_{r}\right\}$ and by applying Theorem 2.7, we obtain that there is $\mathrm{n} \in \mathbb{N}^{d}$ which is the smallest solution of the system $(\mathrm{r}, X)$. Now if for instance $n \in[d-1]$ types are missing in the $r$ first trees of $\mathrm{F}$, then we can apply the same arguments by replacing $d$ by $d-n$ in Theorem 2.7.

Then part 3. is a consequence of part 2. For each $r \geq 1$, we may associate a unique forest to $Y$ with $r$ trees. Since $r$ can be arbitrarily large, the result is proved.

In the same spirit as in [15], the Lukasiewicz-Harris type coding that is displayed in Theorem 3.1 might be used to obtain invariance principles, for any functional that can be encoded simply enough. Besides this result should provide a way to obtain a proper definition of continuous multitype branching trees and forests. Actually, it is natural to think that the latter objects are coded by $d$ independent, $d$-dimensional Lévy processes, with $d-1$ increasing coordinates and a spectrally positive coordinate.

Now we are going to apply our coding of multitype branching forests to the law of their total progeny and give a proof of Theorem 1.2. To that aim, we first need to establish the crucial combinatorial Lemma 3.3. Let $E$ be $\mathbb{Z}_{+}$or a finite integer interval of the type $\{0,1, \ldots, m\}$, with $m \geq 1$ and let $g: E \rightarrow \mathbb{Z}^{d}$, be any application such that $g(0)=0$. For $n \in E$ such that $n \geq 1$, the $n$-cyclical permutations of $g$ are the $n$ applications $g_{q, n}$, $q=0, \ldots, n-1$ which are defined on $E$ by:

$$
g_{q, n}(h) \stackrel{\text { (def })}{=} \begin{cases}g(q+h)-g(q) & \text { if } 0 \leq h \leq n-q, \\ g(h-(n-q))+g(n)-g(q) & \text { if } n-q \leq h \leq n \\ g(h) & \text { if } h \geq n .\end{cases}
$$

Note that $g_{0, n} \equiv g$. The transformation $g \mapsto g_{q, n}$ consists in inverting the parts $\{g(h), 0 \leq$ $h \leq q\}$ and $\{g(h), q \leq h \leq n\}$ in such a way that the new application, $g_{q, n}$, has the same values as $g$ at 0 and $n$, i.e. $g_{q, n}(0)=0$ and $g_{q, n}(n)=g(n)$.

Let $x \in S_{d}$, with finite length $\mathrm{n}=\left(n_{1}, \ldots, n_{d}\right) \in \mathbb{N}^{d}$ and recall the notation $x^{(i)}=$ $\left(x^{i, 1}, \ldots, x^{i, d}\right)$ from Definition 2.1. Then we define the n-cyclical permutations of $x$ by

$$
x_{\mathrm{q}, \mathrm{n}}:=\left(x_{q_{1}, n_{1}}^{(1)}, \ldots, x_{q_{d}, n_{d}}^{(d)}\right), \quad \text { for all } \mathrm{q}=\left(q_{1}, \ldots, q_{d}\right) \leq \mathrm{n}-1_{d},
$$

where we have set $1_{d}=(1,1, \ldots, 1)$. Each sequence $x_{\mathrm{q}, \mathrm{n}}$ will simply be called a cyclical permutation of $x$. Note that there are $n_{1} n_{2} \ldots n_{d}$, cyclical permutations of $x$. Let $\mathrm{r}=$ $\left(r_{1}, \ldots, r_{d}\right) \in \mathbb{Z}_{+}^{d}$ be such that $\mathrm{r}>0$ and assume that $\mathrm{n}$ is a solution of the system $(\mathrm{r}, x)$. 
Then note that $\mathrm{n}$ is also a solution of the system $\left(\mathrm{r}, x_{\mathrm{q}, \mathrm{n}}\right)$, for all $\mathrm{q} \leq \mathrm{n}-1_{d}$, that is,

$$
r_{j}+\sum_{i=1}^{d} x_{\mathrm{q}, \mathrm{n}}^{i, j}\left(n_{i}\right)=0, j=1, \ldots, d .
$$

This remark raises the question of the number of cyclical permutations $x_{\mathrm{q}, \mathrm{n}}$ of $x$, such that $\mathrm{n}$ is the smallest solution of the system $\left(\mathrm{r}, x_{\mathrm{q}, \mathrm{n}}\right)$.

Definition 3.2. Let $x \in S_{d}$, with finite length $\mathrm{n}=\left(n_{1}, \ldots, n_{d}\right) \in \mathbb{N}^{d}$. Let $\mathrm{r}=\left(r_{1}, \ldots, r_{d}\right) \in$ $\mathbb{Z}_{+}^{d}$ be such that $\mathrm{r}>0$ and assume that $\mathrm{n}$ is a solution of the system $(\mathrm{r}, x)$. For $\mathrm{q} \leq \mathrm{n}-1_{d}$, we say that $x_{\mathrm{q}, \mathrm{n}}$ is a good $\mathrm{n}$-cyclical permutation of $x$ with respect to $\mathrm{r}$, if $\mathrm{n}$ is the smallest solution of the system $\left(\mathrm{r}, x_{\mathrm{q}, \mathrm{n}}\right)$, that is $x_{\mathrm{q}, \mathrm{n}} \in S_{d}^{\mathrm{r}}$. When no confusion is possible, we will simply say that $x_{\mathrm{q}, \mathrm{n}}$ is a good cyclical permutation of $x$.

Here is our crucial combinatorial cyclic lemma.

Lemma 3.3 (Multivariate Cyclic Lemma). Let $x \in S_{d}$, with length $\mathrm{n}=\left(n_{1}, \ldots, n_{d}\right) \in \mathbb{N}^{d}$ and let $\mathrm{r}=\left(r_{1}, \ldots, r_{d}\right) \in \mathbb{Z}_{+}^{d}$ be such that $\mathrm{r}>0$. Assume that $\mathrm{n}$ is a solution of the system $(\mathrm{r}, x)$ such that $x^{i, i}\left(n_{i}\right) \neq 0$, for all $i \in[d]$. Then the number of good cyclical permutations of $x$ is $\operatorname{det}\left(\left(-x^{i, j}\left(n_{i}\right)\right)_{i, j \in[d]}\right)$.

This lemma will be proved in Section 4. It is the essential argument for the proof of the following extension of the Ballot Theorem.

Theorem 3.4 (Multivariate Ballot Theorem). Let $Y=\left(Y^{(1)}, \ldots, Y^{(d)}\right)$ be a stochastic process defined on $(\Omega, \mathcal{G}, P)$, with $Y^{(i)}=\left(Y^{i, j}(h), j \in[d], h \geq 0\right), i \in[d]$ and $Y_{0}=0$. We assume that the coordinates $Y^{i, j}$, for $i \neq j$ are $\mathbb{Z}_{+}$valued, nondecreasing and that the coordinates $Y^{i, i}$ are $\mathbb{Z}$ valued and downward skip free. Fix $\mathrm{n}=\left(n_{1}, \ldots, n_{d}\right) \in \mathbb{N}^{d}$, then we assume further that the process $Y$ is n-cyclically exchangeable, that is for any $\mathrm{q}=\left(q_{1}, \ldots, q_{d}\right) \in \mathbb{Z}_{+}^{d}$ such that $\mathrm{q} \leq \mathrm{n}-1_{d}$,

$$
Y_{\mathrm{q}, \mathrm{n}} \stackrel{(\text { law })}{=} Y,
$$

where $Y_{\mathrm{q}, \mathrm{n}}$ is defined as in (3.16) for deterministic functions. Then for any $\mathrm{r}=\left(r_{1}, \ldots, r_{d}\right) \in$ $\mathbb{Z}_{+}^{d}$ such that $\mathrm{r}>0$ and $k_{i j}, i, j \in[d]$, such that $k_{i j} \in \mathbb{Z}_{+}$, for $i \neq j$ and $-k_{j j}=$ $r_{j}+\sum_{i \neq j} k_{i j}$,

$$
\begin{aligned}
& P\left(Y_{n_{i}}^{i, j}=k_{i j}, i, j \in[d] \text { and } \mathrm{n} \text { is the smallest solution of }(\mathrm{r}, Y)\right) \\
= & \frac{\operatorname{det}\left(-k_{i j}\right)}{n_{1} n_{2} \ldots n_{d}} P\left(Y_{n_{i}}^{i, j}=k_{i j}, i, j \in[d]\right) .
\end{aligned}
$$

Proof. If $P\left(Y_{n_{i}}^{i, j}=k_{i j}, i \neq j\right)=0$, then the result is clearly true. Suppose that it is not the case and let $y=\left(y^{(1)}, \ldots, y^{(d)}\right)$ be a deterministic function such that for all $i, j \in[d]$, $y^{i, j}$ is defined on $\mathbb{Z}_{+}, y^{i, j}(0)=0, y^{i, j}\left(n_{i}\right)=k_{i j}$ and

$$
\left.P\left(\left(Y^{(i)}(h), 0 \leq h \leq n_{i}\right)\right)=\left(y^{(i)}(h), 0 \leq h \leq n_{i}\right)\right)>0 .
$$

For $\mathrm{h}=\left(h_{1}, \ldots, h_{d}\right) \in \mathbb{Z}_{+}^{d}$, we set $Y(\mathrm{~h}):=\left(Y^{(1)}\left(h_{1}\right), \ldots, Y^{(d)}\left(h_{d}\right)\right)$ and for $0 \leq \mathrm{q} \leq \mathrm{n}-1_{d}$, using the notation of (3.16), we set $y_{\mathrm{q}, \mathrm{n}}(\mathrm{h}):=\left(y_{q_{1}, n_{1}}^{(1)}\left(h_{1}\right), \ldots, y_{q_{d}, n_{d}}^{(d)}\left(h_{d}\right)\right)$. Let us consider the set

$$
E_{y, \mathrm{n}}=\left\{\left(y_{\mathrm{q}, \mathrm{n}}(\mathrm{h}), 0 \leq \mathrm{h} \leq \mathrm{n}\right): 0 \leq \mathrm{q} \leq \mathrm{n}-1\right\},
$$

of n-cyclical permutations of $y$ over the multidimensional interval $[0, \mathrm{n}]$. Then $\operatorname{Card}\left(E_{y, \mathrm{n}}\right)=$ $n_{1} n_{2} \ldots n_{d}$ and since $Y=\left(Y^{(1)}, \ldots, Y^{(d)}\right)$ is a cyclically exchangeable chain, the law of 
$(Y(\mathrm{~h}), 0 \leq \mathrm{h} \leq \mathrm{n})$, conditionally to the set $\left\{(Y(\mathrm{~h}), 0 \leq \mathrm{h} \leq \mathrm{n}) \in E_{y, \mathrm{n}}\right\}$ is the uniform law in the set $E_{y, \mathrm{n}}$. Moreover, assume that $k_{i i} \neq 0$ for all $i \in[d]$, then conditionally to the set $\left\{(Y(\mathrm{~h}), 0 \leq \mathrm{h} \leq \mathrm{n}) \in E_{y, \mathrm{n}}\right\}$, from Lemma 3.3, the number of good cyclical permutations of $(Y(\mathrm{~h}), 0 \leq \mathrm{h} \leq \mathrm{n})$ is $\operatorname{det}\left(-k_{i j}\right)$. Therefore,

$$
\begin{aligned}
& P\left(Y_{n_{i}}^{i, j}=k_{i j}, i, j \in[d] \text { and } \mathrm{n}\right. \text { is the } \\
& \left.\quad \text { smallest solution of }(\mathrm{r}, Y) \mid(Y(\mathrm{~h}), 0 \leq \mathrm{h} \leq \mathrm{n}) \in E_{y, \mathrm{n}}\right)=\frac{\operatorname{det}\left(-k_{i j}\right)}{n_{1} n_{2} \ldots n_{d}} .
\end{aligned}
$$

Then we obtain the result by summing the identity

$$
\begin{aligned}
& P\left(Y_{n_{i}}^{i, j}=k_{i j}, i, j \in[d]\right. \text { and } \\
& \left.\mathrm{n} \text { is the smallest solution of }(\mathrm{r}, Y),(Y(\mathrm{~h}), 0 \leq \mathrm{h} \leq \mathrm{n}) \in E_{y, \mathrm{n}}\right) \\
= & \frac{\operatorname{det}\left(-k_{i j}\right)}{n_{1} n_{2} \ldots n_{d}} P\left((Y(\mathrm{~h}), 0 \leq \mathrm{h} \leq \mathrm{n}) \in E_{y, \mathrm{n}}\right),
\end{aligned}
$$

over all functions $y$ satisfying (3.18) and with different sets $E_{y, \mathrm{n}}$ of cyclical permutations. Finally, if $k_{i i}=0$, for some $i \in[d]$, then since $n_{i} \geq 1$, we can see that both members of identity (3.17) are equal to 0.

Proof of Theorem 1.2: Let $\mathrm{r}, \mathrm{n}$ and $k_{i j}$ be as in the statement. Let $\mathrm{F}$ be a $d$-type branching forest with progeny law $\nu$, as defined at the beginning of this section and such that the $r$ first trees have root type sequence $\left(c_{1}, \ldots, c_{r}\right) \in C_{d}^{\mathrm{r}}$. Let $X$ be the coding random walk of $\mathrm{F}$, as defined in (3.13). Recall the notation of Theorem 1.2, then from the coding of Subsection 2.2 and Theorem 3.1, we may check that

$$
\begin{aligned}
& \mathbb{P}_{\mathrm{r}}\left(O_{1}=n_{1}, \ldots, O_{d}=n_{d}, A_{i j}=k_{i j}, i, j \in[d], i \neq j\right) \\
= & \mathbf{P}_{\mathrm{c}}\left(X_{n_{i}}^{i, j}=k_{i j}, i, j \in[d] \text { and } \mathrm{n} \text { is the smallest solution of }(\mathrm{r}, X)\right) .
\end{aligned}
$$

Assume first that $\mathrm{n} \in \mathbb{N}^{d}$, then since $X$ is clearly cyclically exchangeable in the sense of Theorem 3.4, we obtain by applying this theorem,

$$
\begin{aligned}
& \mathbf{P}_{\mathrm{c}}\left(X_{n_{i}}^{i, j}=k_{i j}, i, j \in[d] \text { and } \mathrm{n} \text { is the smallest solution of }(\mathrm{r}, X)\right) \\
= & \frac{\operatorname{det}\left(-k_{i j}\right)}{n_{1} n_{2} \ldots n_{d}} P\left(X_{n_{i}}^{i, j}=k_{i j}, i, j \in[d]\right) .
\end{aligned}
$$

On the other hand, since from Theorem 3.1, the random walks $X^{(i)}, i \in[d]$ are independent, we have

$$
\begin{aligned}
& \mathbf{P}_{\mathrm{c}}\left(X_{n_{i}}^{i, j}=k_{i j}, i, j \in[d] \text { and } \mathrm{n} \text { is the smallest solution of }(\mathrm{r}, X)\right) \\
= & \frac{\operatorname{det}\left(-k_{i j}\right)}{n_{1} n_{2} \ldots n_{d}} \prod_{i=1}^{d} P\left(X_{n_{i}}^{i, j}=k_{i j}, j \in[d]\right) .
\end{aligned}
$$

Then from the expression of the law of $X$ given in Theorem 3.1, we obtain

$$
\begin{aligned}
& \mathbf{P}_{\mathrm{c}}\left(X_{n_{i}}^{i, j}=k_{i j}, i, j \in[d] \text { and } \mathrm{n} \text { is the smallest solution of }(\mathrm{r}, X)\right) \\
= & \frac{\operatorname{det}\left(-k_{i j}\right)}{n_{1} n_{2} \ldots n_{d}} \prod_{i=1}^{d} \nu_{i}^{* n_{i}}\left(k_{i 1}, \ldots, k_{i(i-1)}, n_{i}+k_{i i}, k_{i(i+1)}, \ldots, k_{i d}\right),
\end{aligned}
$$


and the result is proved in this case.

Now with no loss of generality, let us assume that for some $0<d^{\prime}<d$, we have $n_{1}, \ldots, n_{d^{\prime}} \in \mathbb{N}$ and $n_{d^{\prime}+1}=\cdots=n_{d}=0$. We point out that from the assumption $n_{j} \geq-k_{j j}=r_{j}+\sum_{i \neq j} k_{i j}$, in this case we necessarily have $r_{j}=0$ and $k_{i j}=0$, for all $i \in[d]$ and $j=d^{\prime}+1, \ldots, d$. Then provided we also have $k_{i j}=0$, for all $i=d^{\prime}+1, \ldots, d$ and $j \in[d], i \neq j$,

$$
\begin{aligned}
& \mathbf{P}_{\mathrm{c}}\left(X_{n_{i}}^{i, j}=k_{i j}, d^{\prime}+1 \leq i \leq d, j \in[d]\right) \\
= & \prod_{i=d^{\prime}+1}^{d} \nu_{i}^{* n_{i}}\left(k_{i 1}, \ldots, k_{i(i-1)}, n_{i}+k_{i i}, k_{i(i+1)}, \ldots, k_{i d}\right)=1 .
\end{aligned}
$$

Define the chain $X$ restricted to $\mathbb{Z}^{d^{\prime}}$, by $X^{\prime}=\left(X^{\prime(1)}, \ldots, X^{\prime\left(d^{\prime}\right)}\right)$, where $X^{\prime(i)}=\left(X^{i, 1}, \ldots, X^{i, d^{\prime}}\right)$, $i \in\left[d^{\prime}\right]$. Set also $\mathrm{n}^{\prime}=\left(n_{1}, \ldots, n_{d^{\prime}}\right)$ and $\mathrm{r}^{\prime}=\left(r_{1}, \ldots, r_{d^{\prime}}\right)$. Then under our assumption on the integers $k_{i j}$, the following identity is satisfied,

$$
\begin{aligned}
& \left\{X_{n_{i}}^{i, j}=k_{i j}, i, j \in[d] \text { and } \mathrm{n} \text { is the smallest solution of }(\mathrm{r}, X)\right\} \\
= & \left\{X_{n_{i}}^{i, j}=k_{i j}, i, j \in\left[d^{\prime}\right] \text { and } \mathrm{n}^{\prime} \text { is the smallest solution of }\left(\mathrm{r}^{\prime}, X^{\prime}\right)\right. \text { and } \\
& \left.X_{n_{i}}^{i, j}=0,1 \leq i \leq d^{\prime}, d^{\prime}+1 \leq j \leq d\right\},
\end{aligned}
$$

so that identity (3.19) can be rewritten as,

$$
\begin{aligned}
& \mathbb{P}_{\mathrm{r}}\left(O_{1}=n_{1}, \ldots, O_{d}=n_{d}, A_{i j}=k_{i j}, i, j \in[d], i \neq j\right) \\
= & \mathbf{P}_{\mathrm{c}}\left(X_{n_{i}}^{i, j}=k_{i j}, i, j \in\left[d^{\prime}\right] \text { and } \mathrm{n}^{\prime} \text { is the smallest solution of }\left(\mathrm{r}^{\prime}, X^{\prime}\right)\right. \text { and } \\
& \left.X_{n_{i}}^{i, j}=0,1 \leq i \leq d^{\prime}, d^{\prime}+1 \leq j \leq d\right) .
\end{aligned}
$$

Moreover, conditionally on the set

$$
\left\{X_{n_{i}}^{\prime i, j^{\prime}}=k_{i j^{\prime}}, X_{n_{i}}^{i, j}=0, i, j^{\prime} \in\left[d^{\prime}\right], d^{\prime}+1 \leq j \leq d\right\},
$$

the chain $X^{\prime}$ is cyclically exchangeable, so that we can conclude in the same way as above that

$$
\begin{aligned}
& \mathbb{P}_{\mathrm{r}}\left(O_{1}=n_{1}, \ldots, O_{d}=n_{d}, A_{i j}=k_{i j}, i, j \in[d], i \neq j\right) \\
= & \frac{\operatorname{det}\left(\left(-k_{i j}\right)_{1 \leq i, j \leq d^{\prime}}\right)}{n_{1} n_{2} \ldots n_{d^{\prime}}} \prod_{i=1}^{d^{\prime}} \nu_{i}^{* n_{i}}\left(k_{i 1}, \ldots, k_{i(i-1)}, n_{i}+k_{i i}, k_{i(i+1)}, \ldots, k_{i d}\right) \\
= & \frac{\operatorname{det}\left(\left(-k_{i j}\right)_{1 \leq i, j \leq d^{\prime}}\right)}{n_{1} n_{2} \ldots n_{d^{\prime}}} \prod_{i=1}^{d} \nu_{i}^{* n_{i}}\left(k_{i 1}, \ldots, k_{i(i-1)}, n_{i}+k_{i i}, k_{i(i+1)}, \ldots, k_{i d}\right) .
\end{aligned}
$$

Finally, if $k_{i j} \neq 0$, for some $i=d^{\prime}+1, \ldots, d$ and $j \in[d]$, then the first and the third members of the above equality are equal to 0 . So the proof is complete.

\section{Proof of Lemmas 2.2 and 3.3}

Proof of Lemma 2.2. Assume that there is a solution $\mathrm{s}=\left(s_{1}, \ldots, s_{d}\right)$ to the system $(\mathrm{r}, x)$, that is: $r_{j}+\sum_{i=1}^{d} x^{i, j}\left(s_{i}\right)=0, j \in[d]$. Let us write this equation in the following 
form:

$$
r_{j}+\sum_{i \neq j} x^{i, j}\left(s_{i}\right)+x^{j, j}\left(s_{j}\right)=0, \quad j=1, \ldots, d .
$$

Then recall that for fixed $j$, when the $k_{i}$ 's increase, the term $\sum_{i \neq j} x^{i, j}\left(k_{i}\right)$ increases and when $k_{j}$ increases, the term $x^{j, j}\left(k_{j}\right)$ may decrease only by jumps of amplitude -1 .

For $\mathrm{k}=\left(k_{1}, \ldots, k_{d}\right)=0$, we have $r_{j}+\sum_{i \neq j} x^{i, j}\left(k_{i}\right)+x^{j, j}\left(k_{j}\right)=r_{j}, j \in[d]$. So for the left hand side of the later equation to reach 0 , each $k_{i}$ has to be at least $\tau^{(i)}\left(r_{i}\right)$, where $\tau^{(i)}$ has been defined in (2.9). (In this proof, we found more convenient to use the notation $\tau^{(i)}(k)$ for $\tau_{k}^{(i)}$.) Then either

$$
r_{j}+\sum_{i \neq j} x^{i, j}\left(\tau^{(i)}\left(r_{i}\right)\right)+x^{j, j}\left(\tau^{(j)}\left(r_{j}\right)\right)=0, \quad j=1, \ldots, d,
$$

or all of the terms $r_{j}+\sum_{i \neq j} x^{i, j}\left(\tau^{(i)}\left(r_{i}\right)\right)+x^{j, j}\left(\tau^{(j)}\left(r_{j}\right)\right), j \in[d]$ are greater or equal than 0 , at least one of them being strictly greater than 0 .

Then in the latter case, for $r_{j}+\sum_{i \neq j} x^{i, j}\left(k_{i}\right)+x^{j, j}\left(k_{j}\right)$ to attain 0 , each of the $k_{j}$ 's has to be at least $\tau^{(j)}\left(r_{j}+\sum_{i \neq j} x^{i, j}\left(\tau^{(i)}\left(r_{i}\right)\right)\right)$. This argument can be repeated until all of the terms $r_{j}+\sum_{i \neq j} x^{i, j}\left(k_{i}\right)+x^{j, j}\left(k_{j}\right)$ attain 0 . More specifically, set $v_{1}^{(j)}=r_{j}$ and for $n \geq 1$,

$$
v_{n+1}^{(j)}=r_{j}+\sum_{i \neq j} x^{i, j}\left(\tau^{(i)}\left(v_{n}^{(i)}\right)\right)
$$

and set $k_{j}^{(n)}=\tau^{(j)}\left(v_{n}^{(j)}\right)$. For $n \geq 1$, either

$$
r_{j}+\sum_{i \neq j} x^{i, j}\left(k_{i}^{(n)}\right)+x^{j, j}\left(k_{j}^{(n)}\right)=0, \quad j=1, \ldots, d,
$$

or all of the terms $r_{j}+\sum_{i \neq j} x^{i, j}\left(k_{i}^{(n)}\right)+x^{j, j}\left(k_{j}^{(n)}\right), j \in[d]$ are greater or equal than 0 , at least one of them being strictly greater than 0 . In the later case, for all of the terms $r_{j}+\sum_{i \neq j} x^{i, j}\left(k_{i}\right)+x^{j, j}\left(k_{j}\right), j \in[d]$ to vanish, the index $\mathrm{k}$ has to be at least $\mathrm{k}^{(n+1)}=\left(k_{1}^{(n+1)}, \ldots, k_{d}^{(n+1)}\right)$. But since there is a solution s to the equation $(\mathrm{r}, x)$, there is necessarily a finite index $n_{0}$ such that $\mathrm{k}^{\left(n_{0}\right)} \leq \mathrm{s}$ and

$$
r_{j}+\sum_{i \neq j} x^{i, j}\left(k_{i}^{\left(n_{0}\right)}\right)+x^{j, j}\left(k_{j}^{\left(n_{0}\right)}\right)=0 .
$$

That is, for all $j \in[d], k_{j}^{\left(n_{0}\right)}=\tau^{(j)}\left(r_{j}+\sum_{i \neq j} x^{i, j}\left(k_{i}^{\left(n_{0}\right)}\right)\right)$. Hence $\mathrm{k}^{\left(n_{0}\right)}$ is the smallest solution of the system $(\mathrm{r}, x)$. Moreover by definition, $k_{i}^{\left(n_{0}\right)}=\min \left\{n: x_{n}^{i, i}=\min _{0 \leq k \leq k_{i}^{\left(n_{0}\right)}} x_{k}^{i, i}\right\}$. This proves the first part of the lemma.

Let $\mathrm{r}^{\prime}=\left(r_{1}^{\prime}, \ldots, r_{d}^{\prime}\right) \in \mathbb{Z}_{+}^{d}$ be such that $\mathrm{r}^{\prime} \leq \mathrm{r}$. Then we prove that there is a smallest solution to the system $\left(\mathrm{r}^{\prime}, x\right)$ similarly. More specifically, since there is a smallest solution to the equation

$$
r_{j}^{\prime}+\sum_{i \neq j} x^{i, j}\left(s_{i}\right)+x^{j, j}\left(s_{j}\right)=r_{j}^{\prime}-r_{j} \leq 0, \quad j=1, \ldots, d,
$$


then by the same arguments as in the first part, we prove that there is a smallest solution to the equation

$$
r_{j}^{\prime}+\sum_{i \neq j} x^{i, j}\left(s_{i}\right)+x^{j, j}\left(s_{j}\right)=0, \quad j=1, \ldots, d .
$$

Let $\mathrm{k}$ and $\mathrm{k}^{\prime}$ be respectively the smallest solutions of $(\mathrm{r}, x)$ and $\left(\mathrm{r}^{\prime}, x\right)$. Then we have,

$$
r_{j}-r_{j}^{\prime}+r_{j}^{\prime}+\sum_{i=1}^{d} x^{i, j}\left(k_{i}^{\prime}\right)+\sum_{i=1}^{d} \tilde{x}^{i, j}\left(k_{i}-k_{i}^{\prime}\right)=0 .
$$

Since $r_{j}^{\prime}+\sum_{i=1}^{d} x^{i, j}\left(k_{i}^{\prime}\right)=0$, the above equation shows that $\mathrm{k}-\mathrm{k}^{\prime}$ is a solution of the system $\left(\mathrm{r}-\mathrm{r}^{\prime}, \tilde{x}\right)$. Moreover, if $\mathrm{k}^{\prime \prime}$ was a strictly smaller solution of $\left(\mathrm{r}-\mathrm{r}^{\prime}, \tilde{x}\right)$, than $\mathrm{k}-\mathrm{k}^{\prime}$ (i.e. $\mathrm{k}^{\prime \prime}<\mathrm{k}-\mathrm{k}^{\prime}$ ) then we would have

$$
\begin{aligned}
r_{j}-r_{j}^{\prime}+r_{j}^{\prime}+\sum_{i=1}^{d} x^{i, j}\left(k_{i}^{\prime}\right)+\sum_{i=1}^{d} \tilde{x}^{i, j}\left(k_{i}^{\prime \prime}\right) & =r_{j}+\sum_{i=1}^{d} x^{i, j}\left(k_{i}^{\prime}+k_{i}^{\prime \prime}\right) \\
& =0,
\end{aligned}
$$

so that $\mathrm{k}^{\prime}+\mathrm{k}^{\prime \prime}$ would be a solution of $(\mathrm{r}, x)$, strictly smaller than $\mathrm{k}$, which is a contradiction.

Proof of Lemma 3.3. Recall from Lemma 2.5 that to each forest $\left(\mathbf{f}, c_{\mathbf{f}}\right) \in \overline{\mathcal{F}}_{d}^{\mathrm{r}}$, we can associate a system $(\mathrm{r}, x)$, with smallest solution $\mathrm{k}$, where $x \in \bar{S}_{d}^{\mathrm{r}}$. We then define the Laplacian matrix of each forest $\left(\mathbf{f}, c_{\mathbf{f}}\right) \in \overline{\mathcal{F}}_{d}^{\mathrm{r}}$ by $\left(k_{i j}\right)=\left(x^{i, j}\left(k_{i}\right)\right)$. We will also sometimes say that $\left(k_{i j}\right)$ is the Laplacian matrix of the associated coding sequence $x$. (Actually, in the conventional terminology of graph theory, $\left(k_{i j}\right)$ would rather be the Laplacian matrix of a directed graph on the set of vertices $[d]$, with incidence matrix $U=\left(u_{i j}\right)_{i, j \in[d]}$, where $u_{i j}=k_{i j}$, if $i \neq j$ and $u_{i i}=0, i \in[d]$.) Recall that $-k_{j j}=r_{j}+\sum_{i \neq j} k_{i j}$ and that for $i \neq j, k_{i j}$ is the total number of vertices in $\mathbf{v}(\mathbf{f})$ with type $j$, whose parent has type $i$ and that $-k_{i i}$ is the total number of vertices of type $i$ in $\mathbf{v}(\mathbf{f})$.

We say that integers $a_{1}, \ldots, a_{n}$ are ranked in the increasing order, up to a cyclical permutation, if there is a cyclical permutation $\sigma$ of the set $[n]$, such that $a_{\sigma(1)}, \ldots, a_{\sigma(n)}$ are ranked in the increasing order.

Definition 4.1. Let $\mathrm{r} \in \mathbb{Z}_{+}^{d}$ be such that $\mathrm{r}>0$.

1. An element $\left(\mathbf{f}, c_{\mathbf{f}}\right)$ of $\overline{\mathcal{F}}_{d}^{\mathrm{r}}$ is said to be a simple forest if for each type $i \in[d]$, at most one vertex of type $i$ in $\mathbf{v}(\mathbf{f})$ has children (the others are leaves). To each vertex of type $i$, we associate an integer in $\left[n_{i}\right]$ which is called its label. Then for each $i$, the sequence of appearance of labels of vertices of type $i$ in the breadth first search of $\left(\mathbf{f}, c_{\mathbf{f}}\right)$ is ranked in the increasing order, up to a cyclical permutation, see Figure 6.

2. For $x \in \bar{S}_{d}^{\mathrm{r}}$, the system $(\mathrm{r}, x)$, with smallest solution $\mathrm{k}=\left(k_{1}, \ldots, k_{d}\right)$ is called a simple system if for all $i \in[d]$, there is $k_{i}^{\prime} \leq k_{i}-1$, such that for all $k=0, \ldots, k_{i}^{\prime}$ and $i \neq j, x^{i, j}(k)=0$ and for all $k=\left(k_{i}^{\prime}+1\right) \vee k_{i}, \ldots, k_{i}$ and $i \neq j, x^{i, j}(k)=$ $x^{i, j}\left(k_{i}\right)$.

In other words, simple systems are elements $x \in \bar{S}_{d}^{\mathrm{r}}$ such that for all $i \in[d]$, the sequences $x^{i, j}, j \in[d]$ have at most one positive jump that occurs at the same point in $\left\{0, \ldots, k_{i}\right\}$. Then we have the following straightforward result. 
Proposition 4.2. If a forest is simple, then its associated system (as defined in Lemma 2.5) is simple.

Definition 4.3. An elementary forest is a forest of $\mathcal{F}_{d}$ that contains exactly one vertex of each type. In particular, each elementary forest contains exactly d vertices and is coded by the $d$ couples $\left(j_{i}, i\right), i \in[d]$, where $j_{i}$ is the type of the parent of the vertex of type $i$. If the vertex of type $i$ is a root, then we set $j_{i}=0$. We define the set $D$ of vectors $\left(j_{1}, \ldots, j_{d}\right)$, $0 \leq j_{i} \leq d$ such that $\left(j_{i}, i\right), i \in[d]$ codes an elementary forest.

Lemma 4.4. Let $\mathrm{r}=\left(r_{1}, \ldots, r_{d}\right) \in \mathbb{Z}_{+}^{d}$ such that $\mathrm{r}>0$ and let $(\mathrm{r}, x)$ be a simple system with smallest solution $\mathrm{k}=\left(k_{1}, \ldots, k_{d}\right) \in \mathbb{N}^{d}$, then the number of good cyclical permutations of $x$ is

$$
\sum_{\left(j_{1}, \ldots, j_{d}\right) \in D} \prod_{i=1}^{d} k_{j_{i} i},
$$

where for $i, j \in[d], k_{i j}=x^{i, j}\left(k_{i}\right)$ and where we have set $k_{0 i}=r_{i}$.

Proof. The proof will be performed by reasoning on forests. Let $(\mathrm{r}, x)$ be as in the statement. From Lemma 2.5 we can associate to $(\mathrm{r}, x)$ a forest $\left(\mathbf{f}, c_{\mathbf{f}}\right)$ of $\overline{\mathcal{F}}_{d}^{\mathrm{r}}$ such that for each type $i \in[d]$, at most one vertex of type $i$ in $\mathbf{v}(\mathbf{f})$ has children (the others are leaves). Then by labeling all the vertices of this forest in the breadth for search order, we obtain a simple forest, see Definition 4.1. Note that for each good cyclical permutation $x_{\mathrm{q}, \mathrm{k}}$ of $x$, the system $\left(\mathrm{r}, x_{\mathrm{q}, \mathrm{k}}\right)$ is simple itself and to each one, we may associate a unique simple forest which is obtain by cyclical permutations of the vertices of type $i$ in $\left(\mathbf{f}, c_{\mathbf{f}}\right)$, for each $i$. Conversely, recall Proposition 4.2, then the simple system $(\mathrm{r}, y)$ which is associated to each simple forest with Laplacian matrix $\left(k_{i j}\right)$, through Lemma 2.5 is necessarily obtained from a good cyclical permutation of $x$. Indeed the corresponding sequences $y^{i, j}$, for $i \neq j$, have exactly one jump and are such that $y^{i, j}\left(k_{i}\right)=k_{i j}$. So $y$ is nothing but a cyclical permutation of $x$. These arguments prove that the number of good cyclical permutations of $x$ is equal to the number of simple forests with Laplacian matrix $\left(k_{i j}\right)$.

Then let us prove that the number of simple forests with Laplacian matrix $\left(k_{i j}\right)$ is $\sum_{\left(j_{1}, \ldots, j_{d}\right) \in D} \prod_{i=1}^{d} k_{j_{i} i}$. We make the additional assumption that for each $i$, there is exactly one vertex who has children. Then observe that to each simple forest, we can associate a unique elementary forest in the following way: the vertex of type $j$ in the elementary forest is the parent of the vertex of type $i$ if, in the simple forest the parent of the vertex of type $i$ who has children has type $j$ (recall that $j=0$ if the vertex of type $i$ is a root). An example of an elementary forest associated to a simple forest is given in Figure 6 . Then let $\left(j_{1}, \ldots, j_{d}\right) \in D$. We easily see that the monomial $\prod_{i=1}^{d} k_{j_{i} i}$ is the number of simple forests such that for each $i$, the parent of the vertex of type $i$ who has children has type $j_{i}$. Indeed, there are $k_{j_{i}}$ possibilities to choose the vertex of type $i$ who has children. In other words, $\prod_{i=1}^{d} k_{j_{i} i}$ is the number of all possible simple forests to which we can associate the same elementary forest which is coded by $\left(j_{i}, i\right), i \in[d]$. Then in order to obtain the total number of simple forests with Laplacian matrix $\left(k_{i j}\right)$, it remains to perform the summation of these monomials over all the possible elementary forests. So we obtained the formula of the statement, under our additional assumption.

Then we have proved the result for simple systems such that for all $i \in[d]$, there is $j \neq i$ with $k_{i j}>0$. Now assume that for all $i \in[d-1]$, there is $j \neq i$ such that $k_{i j}>0$ and that $k_{d j}=0$, for all $j \in[d]$. Then in such a system, we have $x^{(d)} \equiv 0$. Let us consider the system $\left(\mathrm{r}^{\prime}, x^{\prime}\right)$, where $\mathrm{r}^{\prime}=\left(r_{1}, \ldots, r_{d-1}\right)$ and $x^{\prime}=\left(x^{(1)}, \ldots, x^{(d-1)}\right)$. From what has just been proved, the number of good cyclical permutation of $\left(\mathrm{r}^{\prime}, x^{\prime}\right)$ is $h_{d-1}:=$ 


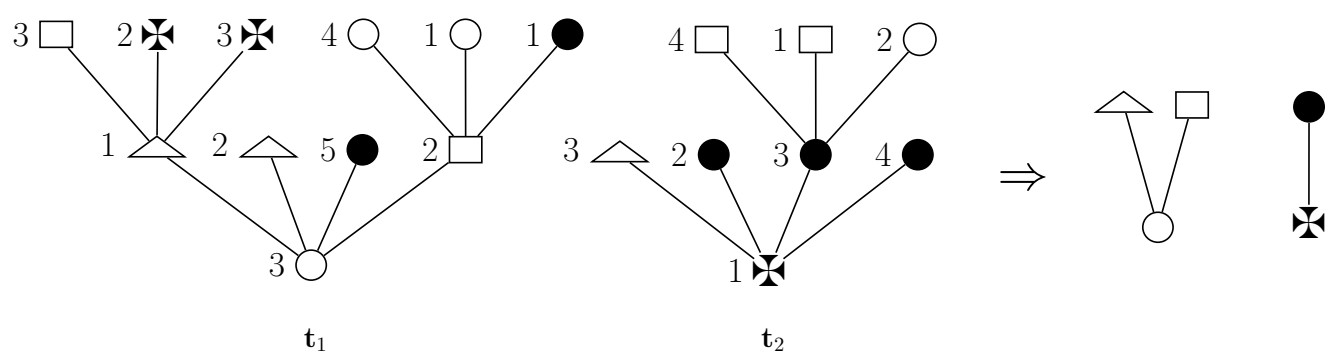

FiguRE 6. A simple forest and its associated elementary forest.

$\sum_{\left(j_{1}, \ldots, j_{d-1}\right) \in D^{\prime}} \prod_{i=1}^{d-1} k_{j_{i} i}$ where $D^{\prime}$ is the set that is defined in Definition 4.3 and where we replaced $d$ by $d-1$. Then in order to obtain all the good cyclical permutations of $x$ it remains to consider the $k_{d}$ cyclical permutations of the sequence $x^{(d)}$. Since the latter are all identical, we have actually $k_{d} \times h_{d-1}$ good cyclical permutations of $x$. Then we conclude by noticing that $k_{d} \times h_{d-1}=\sum_{\left(j_{1}, \ldots, j_{d}\right) \in D} \prod_{i=1}^{d} k_{j_{i} i}$, since $k_{d}=r_{d}+\sum_{i \neq d} k_{i d}=\sum_{i=0, i \neq d}^{d} k_{i d}$. The general case where $k_{i j}=0$, for all $j \in[d]$, for other types $i$ is obtained in the same manner.

Lemma 4.5. For any $\mathrm{r} \in \mathbb{Z}_{+}^{d}$ and any integer valued matrix $\left(k_{i j}\right)_{i, j \in[d]}$, such that $k_{i j}$ is nonnegative for $i \neq j$ and $-k_{j j}=r_{j}+\sum_{i \neq j} k_{i j}, j \in[d]$,

$$
\operatorname{det}\left(-k_{i j}\right)=\sum_{\left(j_{1}, \ldots, j_{d}\right) \in D} \prod_{i=1}^{d} k_{j_{i} i},
$$

where the set $D$ is defined in Definition 4.3 and $k_{0 i}=r_{i}$.

Proof. The proof is a direct consequence of the matrix tree theorem for directed graphs, due to Tutte [23], see Section 3.6, page 470 therein. However, Theorem 3.1 in [18] that implies Tutte's theorem is actually easier to apply, since it uses a setting which is closer to ours. Let us consider a set $\left\{v_{0}, v_{1}, \ldots, v_{d}\right\}$ of $d+1$ vertices and in the notations of [18], set $W=L=\left\{v_{0}\right\}$. Then the family $\mathcal{F}_{W, L}$ that is described in Theorem 3.1 of [18] is in bijection with the set of elementary forests, or equivalently with the set $D$, and identity (3.2) in this theorem is exactly $\operatorname{det}\left(-k_{i j}\right)=\sum_{\left(j_{1}, \ldots, j_{d}\right) \in D} \prod_{i=1}^{d} k_{j_{i} i}$.

Lemma 4.6. Let $x \in S_{d}$, with length $\mathrm{k}=\left(k_{1}, \ldots, k_{d}\right) \in \mathbb{N}^{d}$. Let $\mathrm{r}=\left(r_{1}, \ldots, r_{d}\right) \in \mathbb{Z}_{+}^{d}$ be such that $\mathrm{r}>0$ and assume that $\mathrm{k}$ is a solution of the system $(\mathrm{r}, x)$. Assume moreover that $x_{k}^{i, i}=-k$, for all $k=0, \ldots, k_{i}$ and $i \in[d]$. Then the number of good cyclical permutations of $x$ is $\operatorname{det}\left(\left(x^{i, j}\left(k_{i}\right)\right)_{i, j \in[d]}\right)$.

Proof. Let $x$ be as in the statement. If for all $i \in[d]$, the sequences $x^{i, j}, j \in[d]$ have at most one positive jump that occurs at the same point in $\left\{0, \ldots, k_{i}\right\}$, then from Definition 4.1, Lemma 4.4 and Lemma 4.5, there is a cyclical permutation $x^{\prime}$ of $x$ such that $\left(\mathrm{r}, x^{\prime}\right)$ is a simple system and the result follows in this case. In general, let us prove that there is a simple system, with Laplacian matrix $\left(x^{i, j}\left(k_{i}\right)\right)$, and whose number of good cyclical permutations is the same as this of $x$. 
Fix any index $m \in[d]$ and assume without loss of generality, that $m \neq 1$, and

$$
x^{m, 1}\left(k_{m}\right)-x^{m, 1}\left(k_{m}-1\right)>0 .
$$

From $x$, we define a new sequence $\tilde{x} \in S_{d}$ with length $\mathrm{k}$ as follows:

$$
\left\{\begin{array}{l}
\tilde{x}^{m, j}(k)=x^{m, j}(k), \quad j=2, \ldots, d, k=1, \ldots, k_{m}, \\
\tilde{x}^{m, 1}(k)=x^{m, 1}(k), \quad k=1, \ldots, k_{m}-2, k_{m}, \\
\tilde{x}^{m, 1}\left(k_{m}-1\right)=x^{m, 1}\left(k_{m}-1\right)+1 .
\end{array}\right.
$$

All the other coordinates remain unchanged, that is,

$$
\tilde{x}^{i, j}(k)=x^{i, j}(k), \quad i, j=1, \ldots, d, \quad k=1, \ldots, k_{i}, \quad i \neq m .
$$

The sequence $\tilde{x}$ is obtained from $x$ by decreasing by one unit, the last jump of the coordinate $x^{m, 1}$, that is $x^{m, 1}\left(k_{m}\right)-x^{m, 1}\left(k_{m}-1\right)$. (Therefore the jump $x^{m, 1}\left(k_{m}-1\right)-$ $x^{m, 1}\left(k_{m}-2\right)$ is increased by one unit.) Denote by $N_{\mathrm{r}, x}$ the number of good cyclical permutations of $x$. We claim that

$$
N_{\mathrm{r}, \tilde{x}} \geq N_{\mathrm{r}, x}
$$

To achieve this aim, first observe that $\mathrm{k}$ is a solution of the system $(\mathrm{r}, \tilde{x})$, that is

$$
r_{j}+\sum_{i=1}^{d} \tilde{x}^{i, j}\left(k_{i}\right)=0, j=1, \ldots, d,
$$

and note the straightforward inequality,

$$
\tilde{x}^{i, j}(k) \geq x^{i, j}(k), \quad i, j=1, \ldots, d, \quad k=1, \ldots, k_{i} .
$$

Therefore, if $\mathrm{k}$ is the smallest solution of $(\mathrm{r}, x)$ then it is also the smallest solution of $(\mathrm{r}, \tilde{x})$. Moreover, for $\mathrm{q}=\left(q_{1}, \ldots, q_{d}\right) \in \mathbb{N}^{d}$ satisfying $q_{m}<k_{m}-1$, one easily checks that the same inequality holds, that is

$$
\tilde{x}_{\mathrm{q}, \mathrm{k}}^{i, j}(k) \geq x_{\mathrm{q}, \mathrm{k}}^{i, j}(k), \quad i, j=1, \ldots, d, \quad k=1, \ldots, k_{i},
$$

so that if $\mathrm{k}$ is the smallest solution of $\left(\mathrm{r}, x_{\mathrm{q}, \mathrm{k}}\right)$ (i.e. $x_{\mathrm{q}, \mathrm{k}}$ is a good cyclical permutation of $x)$ then it is also the smallest solution of $\left(\mathrm{r}, \tilde{x}_{\mathrm{q}, \mathrm{k}}\right)$.

Now it remains to study the case where $q_{m}=k_{m}-1$. Assume that $x_{\mathrm{q}, \mathrm{k}}$ is a good cyclical permutation of $x$, but that $\tilde{x}_{\mathrm{q}, \mathrm{k}}$ is not a good cyclical permutation of $\tilde{x}$. Then in order to obtain the inequality (4.22), we have to find a good cyclical permutation $\tilde{x}_{1, \mathrm{k}}$ of $\tilde{x}$ such that $x_{1, \mathrm{k}}$ is not a good cyclical permutation of $x$. Let us define the sequence $\hat{x} \in S_{d}$ with length $\mathrm{k}$, which is obtained by decreasing by one unit the first coordinate of $x_{\mathrm{q}, \mathrm{k}}^{(m)}$, that is

$$
\left\{\begin{array}{l}
\hat{x}^{(i)} \equiv x_{\mathrm{q}, \mathrm{k}}^{(i)}, i \neq m, \\
\hat{x}^{(m)}(k)=x_{\mathrm{q}, \mathrm{k}}^{(m)}(k)-\mathbf{e}_{1}, k \geq 1,
\end{array}\right.
$$

where $\mathbf{e}_{1}=(1,0, \cdots, 0)$, is the $d$ dimensional unit vector. Since $\mathrm{k}$ is the smallest solution of $\left(\mathrm{r}, x_{\mathrm{q}, \mathrm{k}}\right)$, then $\mathrm{k}$ is the smallest solution of $\left(\mathrm{r}+\mathbf{e}_{1}, \hat{x}\right)$ by the definition of $\hat{x}$. Moreover, from Lemma $2.2(i i)$, the system $\left(\mathbf{e}_{1}, \hat{x}\right)$ admits a smallest solution which is less than $\mathrm{k}$. Let us call $\mathrm{p}$ this solution. Then $\mathrm{p}>0$ and from Lemma 2.2 (ii), $\mathrm{k}-\mathrm{p}$ is the smallest solution of $\left(\mathrm{r}, \hat{x}_{\mathrm{p}, \mathrm{k}}\right)$.

Then let us consider the cyclical permutation of $\tilde{x}_{\mathrm{q}, \mathrm{k}}$ at $\mathrm{p}$. It is a cyclical permutation of $\tilde{x}$ that we shall denote by $\tilde{x}_{1, \mathrm{k}}$. Note that $\hat{x}$ and $\tilde{x}_{\mathrm{q}, \mathrm{k}}$ only differ from the last jump of 
$\hat{x}^{m, 1}$ and $\tilde{x}_{\mathrm{q}, \mathrm{k}}^{m, 1}$, more specifically, $\tilde{x}_{\mathrm{q}, \mathrm{k}}\left(k_{m}\right)-\tilde{x}_{\mathrm{q}, \mathrm{k}}\left(k_{m}-1\right)=\hat{x}\left(k_{m}\right)-\hat{x}\left(k_{m}-1\right)+1$. Then from the above constructions, we can see that $\tilde{x}_{1, \mathrm{k}}$ is obtained as follows:

$$
\tilde{x}_{1, \mathrm{k}}^{(i)}(k)= \begin{cases}\hat{x}_{\mathrm{p}, \mathrm{k}}^{(i)}(k), & k \leq k_{i}-p_{i}, i \neq m, \\ \hat{x}_{\mathrm{p}, \mathrm{k}}^{(m)}(k), & k<k_{m}-p_{m}, \\ \hat{x}_{\mathrm{p}, \mathrm{k}}^{(i)}\left(k_{i}-p_{i}\right)+\hat{x}^{(i)}\left(k-\left(k_{i}-p_{i}\right)\right), & k_{i}-p_{i} \leq k \leq k_{i}, i \neq m, \\ \hat{x}_{\mathrm{p}, \mathrm{k}}^{(m)}\left(k_{m}-p_{m}\right)+\hat{x}^{(m)}\left(k-\left(k_{m}-p_{m}\right)\right)+\mathbf{e}_{1}, & k_{m}-p_{m} \leq k \leq k_{m} .\end{cases}
$$

Since $\mathrm{k}-\mathrm{p}$ is the smallest solution of $\left(\mathrm{r}, \hat{x}_{\mathrm{p}, \mathrm{k}}\right)$ and since $\tilde{x}_{1, \mathrm{k}}$ is strictly greater than $\hat{x}_{\mathrm{p}, \mathrm{k}}$ at point $\mathrm{k}-\mathrm{p}$, there is no solution to $\left(\mathrm{r}, \tilde{x}_{1, \mathrm{k}}\right)$, on the (multidimensional) interval $[0, \mathrm{k}-\mathrm{p}]$. Moreover, also from the construction of $\left(\mathrm{r}, \tilde{x}_{1, \mathrm{k}}\right)$, since $\mathrm{p}$ is the smallest solution of $\left(\mathbf{e}_{1}, \hat{x}\right)$, the only solution of $\left(\mathrm{r}, \tilde{x}_{1, \mathrm{k}}\right)$ on the interval $[\mathrm{k}-\mathrm{p}, \mathrm{k}]$ is $\mathrm{k}$. Therefore, the smallest solution of $\left(\mathrm{r}, \tilde{x}_{1, \mathrm{k}}\right)$ is $\mathrm{k}$.

On the other hand, note the following identity

$$
\left(x_{1, \mathrm{k}}^{i, j}(k), i, j \in[d], k \in\left[k_{i}-p_{i}\right]\right)=\left(\hat{x}_{\mathrm{p}, \mathrm{k}}^{i, j}(k), i, j \in[d], k \in\left[k_{i}-p_{i}\right]\right),
$$

which can be seen directly from the definition of $\hat{x}$. It follows that $\mathrm{k}-\mathrm{p}$ is the smallest solution of the system $\left(\mathrm{r}, x_{1, \mathrm{k}}\right)$. But since $\mathrm{p}>0$, the sequence $x_{1, \mathrm{k}}$ is not a good permutation of $x$, and the inequality $(4.22)$ is proved.

Let $\mathrm{q}=\left(0, \ldots, 0, k_{m}-1,0 \ldots, 0\right)$, where $k_{m}-1$ is the $m$-th coordinate of $\mathrm{q}$ and set $y:=\tilde{x}_{\mathrm{q}, \mathrm{k}}$, then by applying the same arguments as above to the chain $y$, we obtain that

$$
N_{\mathrm{r}, \tilde{y}} \geq N_{\mathrm{r}, y}=N_{\mathrm{r}, \tilde{x}} \geq N_{\mathrm{r}, x}
$$

with obvious notations. But by reiterating $k_{m}$ times this operation, we obtain again the chain $x$. This shows that equality holds in (4.22), that is $N_{\mathrm{r}, \tilde{x}}=N_{\mathrm{r}, x}$.

Finally, let $z \in S_{d}$ be a chain with length $\mathrm{k}$, such that $z^{i, j}\left(k_{i}\right)=x^{i, j}\left(k_{i}\right)$, for all $i, j \in[d]$, and such that for all $i \in[d]$, the sequences $z^{i, j}, j \in[d]$ have at most one positive jump that occurs at the same point in $\left\{0, \ldots, k_{i}\right\}$. Then it is easy to see that the chain $x$ can be obtained after several cyclical permutations and iterations of the transformation (4.21) applied to $z$, at any coordinate. Therefore $N_{\mathrm{r}, z}=N_{\mathrm{r}, x}$. Assume that there is a good cyclical permutation $z^{\prime}$ of $z$. Then $\left(\mathrm{r}, z^{\prime}\right)$ is a simple system. Therefore, from Lemma 4.4 and Lemma 4.5, $N_{\mathrm{r}, z^{\prime}}=N_{\mathrm{r}, z}=N_{\mathrm{r}, x}=\operatorname{det}\left(x^{i, j}\left(k_{i}\right)\right)$.

Lemma 4.7. Let $x \in S_{d}^{\mathrm{r}}$ with finite length and let $\bar{x}$ be the sequence of $\bar{S}_{d}^{\mathrm{r}}$ which is associated to $x$, as in (2.10). Then $x$ and $\bar{x}$ have the same number of good cyclical permutations.

Proof. Let $\mathrm{k}$ and $\mathrm{n}$ be the respective lengths of $\bar{x}$ and $x$. In particular, we have $n_{i}=\tau_{k_{i}}^{(i)}, i \in[d]$. Let $\mathrm{q} \leq \mathrm{k}-1_{d}$ be such that $\bar{x}_{\mathrm{q}, \mathrm{k}}$ is a good cyclical permutation of $\bar{x}$. Then clearly, there is $\mathrm{p}=\left(p_{1}, \ldots, p_{d}\right) \leq \mathrm{n}-1_{d}$, such that $p_{i}=\tau_{q_{i}}^{(i)}$. Set $y=x_{\mathrm{p}, \mathrm{n}}$ and let us check that

$$
\bar{y}=\bar{x}_{\mathrm{q}, \mathrm{k}} .
$$

Define $\theta^{(i)}(m)=\min \left\{v: x_{p_{i}, n_{i}}^{i, i}(v)=-m\right\}=\min \left\{v: y^{i, i}(v)=-m\right\}$ and let $\tau_{q_{i}, k_{i}}^{(i)}$ be the cyclical permutation of the sequence $\tau^{(i)}$, as defined in (3.15). Then from the construction of $y$, we can check that

$$
\theta^{(i)}(m)=\tau_{q_{i}, k_{i}}^{(i)}(m), \quad m \leq k_{i}
$$


from which we derive (4.23). Moreover, since $\bar{y}$ is a good cyclical permutation of $\bar{x}$, we deduce from Lemma 2.6 that $y$ is a good cyclical permutation of $x$.

Conversely, let $\mathrm{q}<\mathrm{n}-1_{d}$ such that $x_{\mathrm{q}, \mathrm{n}}$ is a good cyclical permutation of $x$. Then from part $(i)$ of Lemma 2.2, we must have $n_{i}=\min \left\{n: x_{q_{i}, n_{i}}^{i, i}(n)=-k_{i}\right\}$. Therefore, there exists $\mathrm{p}<\mathrm{k}$, such that $q_{i}=\tau_{p_{i}}^{(i)}$. Again, by setting $y=x_{\mathrm{q}, \mathrm{n}}$, we check that $\bar{y}=\bar{x}_{\mathrm{p}, \mathrm{k}}$ and we deduce from Lemma 2.6 that $\bar{y}$ is a good cyclical permutation of $\bar{x}$.

Then we end the proof of Lemma 3.3. Let $x \in S_{d}$ be as in this lemma, that is $x$ has finite length $\mathrm{n}=\left(n_{1}, \ldots, n_{d}\right) \in \mathbb{N}^{d}$ and $\mathrm{n}$ is a solution of the system $(\mathrm{r}, x)$, where $\mathrm{r}=$ $\left(r_{1}, \ldots, r_{d}\right) \in \mathbb{Z}_{+}^{d}$ is such that $\mathrm{r}>0$. Let $k_{i}^{\prime}=-\min _{0 \leq n \leq n_{i}} x_{n}^{i, i}$ and set $n_{i}^{\prime}=\tau_{k_{i}^{\prime}}^{(i)}$. Then $\mathrm{n}^{\prime} \leq \mathrm{n}$, so that we can define the cyclical permutation $y=x_{\mathrm{n}^{\prime}, \mathrm{n}}$ of $x$. By construction, the sequence $\bar{y}$ has length $\mathrm{k}$, where $k_{i}=-x^{i, i}\left(n_{i}\right)$ and $\bar{y}^{i, j}\left(k_{i}\right)=x^{i, j}\left(n_{i}\right), i, j \in[d]$. Note that $k_{i} \geq 1$, from the assumption $x^{i, i}\left(n_{i}\right) \neq 0$. Moreover, $\mathrm{k}$ is a solution of the system $(\mathrm{r}, \bar{y})$. So, thanks to Lemma 4.6, the number of good cyclical permutations of $\bar{y}$ is $\operatorname{det}\left(x^{i, j}\left(n_{i}\right)\right)$ and from Lemmas 2.6 and 4.7 this is also the number of good cyclical permutations of $y$, the latter being clearly the number of good cyclical permutations of $x$.

\section{Applications to SOME COMBinatorial FORMUlas}

5.1. Enumeration of multitype forests. We may now derive from the previous results, some enumeration formulas for multitype forests. In all this subsection, $r_{i}, n_{i}$ and $k_{i j}, i, j \in[d]$ will be integers satisfying conditions of Theorem 1.2, that is $r_{i} \geq 0$, $r_{1}+\cdots+r_{d} \geq 1, k_{i j} \geq 0$, for $i \neq j,-k_{j j}=r_{j}+\sum_{i \neq j} k_{i j}$ and $n_{i} \geq-k_{i i}$. We assume moreover that $-k_{i i}>0$, for all $i \in[d]$.

Our first result is an application of Theorem 1.2 which gives the number of plane forests with $n_{i}$ vertices of type $i, r_{i}$ roots of type $i$ and whose corresponding reduced forest has $\left(k_{i j}\right)$ as a Laplacian matrix. It extends the one dimensional case where, the number of the unlabeled forests with $r$ trees and $n$ vertices is $\frac{r}{n}\left(\begin{array}{c}2 n-r-1 \\ n-r\end{array}\right)$.

Theorem 5.1. Let $\mathcal{F}_{d}^{k_{i j}, \mathrm{n}}$ be the subset of plane forests of $\mathcal{F}_{d}$, with $n_{i}$ vertices of type $i, r_{i}$ roots of type $i$ and such that for $i \neq j, k_{i j}$ vertices of type $j$ have a parent of type $i$, then

$$
\left|\mathcal{F}_{d}^{k_{i j}, \mathrm{n}}\right|=\frac{\operatorname{det}\left(-k_{i j}\right)}{n_{1} n_{2} \ldots n_{d}} \prod_{i, j=1}^{d}\left(\begin{array}{c}
n_{i}+k_{i j}^{\prime}-1 \\
k_{i j}^{\prime}
\end{array}\right),
$$

where $k_{i i}^{\prime}=n_{i}+k_{i i}$ and for $i \neq j, k_{i j}^{\prime}=k_{i j}$.

Proof. We use the same arguments as in Section 6 of Pitman [21] where the case $d=1$ is treated. Let $\mathrm{F}$ be a $d$-type branching forest, as defined in Section 3, with progeny law $\nu$ given by

$$
\nu_{i}\left(k_{1}, \cdots, k_{d}\right)=\prod_{j=1}^{d}\left(1-p_{i j}\right)^{k_{j}} p_{i j}, \quad i=1,2, \ldots d,\left(k_{1}, \cdots, k_{d}\right) \in \mathbb{Z}_{+},
$$

where $0<p_{i j}<1, i, j \in[d]$ That is to say, each individual of type $i$ gives birth to children of different types independently, respectively according to the geometric distribution $\mu_{i j}(\cdot)$ 
with parameter $p_{i j}, j \in[d]$. Then

$$
\nu_{i}\left(k_{1}, \cdots, k_{d}\right)=\prod_{j=1}^{d} \mu_{i j}\left(k_{j}\right)
$$

where for any $n \in \mathbb{N}, \mu_{i j}^{* n}(k)=\left(\begin{array}{c}n+k-1 \\ k\end{array}\right)\left(1-p_{i j}\right)^{k} p_{i j}^{n}, k=0,1, \ldots$

Let $\mathrm{p}(u)=\left(p_{i}(u), i \in[d]\right)$ be the increment of the Lukasiewicz-Harris path related to some vertex $u$ of some forest $\mathbf{f}$, as it is defined in (2.7). Recall also from Subsection 2.1 that $c(u)=c_{\mathbf{f}}(u) \in[d]$ is the type of the vertex $u$. Then for any $\mathbf{f} \in \mathcal{F}_{d}^{k_{i j}, \mathrm{n}}$,

$$
\mathbb{P}_{\mathrm{r}}(\mathrm{F}=\mathbf{f})=\prod_{u \in \mathbf{f}} \nu_{c(u)}(\mathrm{p}(u))=\prod_{u \in \mathbf{f}} \prod_{j=1}^{d}\left(1-p_{c(u) j}\right)^{p_{j}(u)} p_{c(u) j}=\prod_{i, j=1}^{d}\left(1-p_{i j}\right)^{k_{i j}^{\prime}} p_{i j}^{n_{i}} .
$$

Since this probability is the same for all the forests $\mathbf{f} \in \mathcal{F}_{d}^{k_{i j}, \mathrm{n}}$, the following conditional distribution is the uniform distribution on $\mathcal{F}_{d}^{k_{i j}, \mathrm{n}}$ :

$$
\mathbb{P}_{\mathrm{r}}\left(\mathrm{F} \in \cdot \mid O(F)=\mathrm{n}, A_{i j}(F)=k_{i j}, i, j \in[d], i \neq j\right) .
$$

But Theorem 1.2 tells us that

$$
\begin{aligned}
& \mathbb{P}_{\mathrm{r}}\left(O_{1}=n_{1}, \ldots, O_{d}=n_{d}, A_{i j}=k_{i j}, i, j \in[d], i \neq j\right) \\
= & \frac{\operatorname{det}\left(-k_{i j}\right)}{n_{1} n_{2} \ldots n_{d}} \prod_{i=1}^{d} \nu_{i}^{* n_{i}}\left(k_{i 1}, \ldots, k_{i(i-1)}, n_{i}+k_{i i}, k_{i(i+1)}, \ldots, k_{i d}\right) \\
= & \frac{\operatorname{det}\left(-k_{i j}\right)}{n_{1} n_{2} \ldots n_{d}} \prod_{i, j=1}^{d} \mu_{i j}^{* n_{i}}\left(k_{i j}^{\prime}\right) \\
= & \frac{\operatorname{det}\left(-k_{i j}\right)}{n_{1} n_{2} \ldots n_{d}} \prod_{i, j=1}^{d}\left(\begin{array}{c}
n_{i}+k_{i j}^{\prime}-1 \\
k_{i j}^{\prime}
\end{array}\right)\left(1-p_{i j}\right)^{k_{i j}^{\prime}} p_{i j}^{n_{i}} \\
= & \frac{\operatorname{det}\left(-k_{i j}\right)}{n_{1} n_{2} \ldots n_{d}} \prod_{i, j=1}^{d}\left(\begin{array}{c}
n_{i}+k_{i j}^{\prime}-1 \\
k_{i j}^{\prime}
\end{array}\right)\left(\prod_{i, j=1}^{d}\left(1-p_{i j}\right)^{k_{i j}^{\prime}} p_{i j}^{n_{i}}\right) .
\end{aligned}
$$

Comparing this probability with (5.24), we obtain our result.

Now we shall enumerated labeled forests according to the degree of their vertices. Some of the next results have recently been obtained.

Definition 5.2. To each forest $\mathbf{f}$ with $n_{i}$ vertices of type $i$ and to each vertex of type $i$ in $\mathbf{f}$, we associate an integer in $\left[n_{i}\right]$, which is called its label. Then $\mathbf{f}$ is called a labeled plane forest. Let $\mathcal{L}$ be the set of labeled plane forests with $n_{i}$ vertices of type $i, r_{i}$ roots of type $i$ and whose corresponding reduced forest has $\left(k_{i j}\right)$ as a Laplacian matrix.

Let $\mathbf{c}=\left(c_{i, j, k}\right)_{i, j \in[d], k \in\left[n_{i}\right]}$ be a tuple of non-negative integers such that $k_{i j}^{\prime}=\sum_{k=1}^{n_{i}} c_{i, j, k}$, where $k_{i j}^{\prime}$ is defined in Theorem 5.1. We will denote by $\mathcal{L}(\mathbf{c})$ the subset of $\mathcal{L}$, of forests in which the vertex of type $i$ with label $k$ has $c_{i, j, k}$ offspring of type $j$. Then $\mathbf{c}$ is called the indegree tuple of the forest $\mathbf{f} \in \mathcal{L}(\mathbf{c})$.

The following result has been obtained in [3], see Proposition 11. 
Proposition 5.3 (Enumeration of labeled plane forests by indegree tuple). For any indegree tuple $\mathbf{c}=\left(c_{i, j, k}\right)_{i, j \in[d], k \in\left[n_{i}\right]}$, the number of forests with $\mathbf{c}$ as the indegree tuple in $\mathcal{L}$ is

$$
|\mathcal{L}(\mathbf{c})|=\frac{\prod_{j=1}^{d}\left(n_{j}-1\right) !}{\prod_{i \in[d]} r_{i} ! \prod_{i, j \in[d], k \in\left[n_{i}\right]} c_{i, j, k} !} \operatorname{det}\left(-k_{i j}\right) .
$$

Proof. Let $\mathbf{f} \in \mathcal{L}(\mathbf{c})$ and let $x$ be its coding sequence, given by Theorem 2.7. According to Lemmas 4.6 and 4.7 , there are $\operatorname{det}\left(-k_{i j}\right)$ good cyclical permutations of $x$, each one coding a different forest in $\mathcal{L}(\mathbf{c})$. On the other hand, any two forests $\mathbf{f}$ and $\mathbf{f}^{\prime}$ of $\mathcal{L}(\mathbf{c})$ are coded through two sequences $x$ and $x^{\prime}$ such that for each $i \in[d]$, the sequence of increments $\left(\Delta x_{1}^{(i)}, \ldots, \Delta x_{n_{i}}^{(i)}\right)$ is a permutation of the sequence of increments $\left(\Delta x_{1}^{\prime(i)}, \ldots, \Delta{x^{\prime}}_{n_{i}}^{(i)}\right)$. Then there are $\prod_{j=1}^{d}\left(n_{j}-1\right)$ ! det $\left(-k_{i j}\right)$ good permutations of $x$, where by good permutation, we mean a sequence $x^{\prime}$ which codes a forest in $\mathcal{L}(\mathbf{c})$ and such that the sequence of increments $\left(\Delta{x^{\prime}}_{1}^{(i)}, \ldots, \Delta{x^{\prime}}_{n_{i}}^{(i)}\right)$ is a permutation of the sequence of increments $\left(\Delta x_{1}^{(i)}, \ldots, \Delta x_{n_{i}}^{(i)}\right)$.

In the enumeration that we have just done, we counted forests $\mathbf{f}, \mathbf{f}^{\prime} \in \mathcal{L}(\mathbf{c})$ such that $\mathbf{f}^{\prime}$ can be obtained by permuting in $\mathbf{f}$ the $c_{i, j, k}$ subtrees whose roots are the $c_{i, j, k}$ children of type $j$ of the $k$ th vertex of type $i$, for some $i, j \in[d]$ and $k \in\left[n_{i}\right]$ or by permuting the trees with the same type roots in the whole forest. But in this case, $\mathbf{f}$ and $\mathbf{f}^{\prime}$ are the same forest. Therefore, we still have to divide the number $\prod_{j=1}^{d}\left(n_{j}-1\right)$ ! $\operatorname{det}\left(-k_{i j}\right)$ by $\prod_{i \in[d]} r_{i} ! \prod_{i, j \in[d], k \in\left[n_{i}\right]} c_{i, j, k} !$, that is

$$
|\mathcal{L}(\mathbf{c})|=\frac{\prod_{j=1}^{d}\left(n_{j}-1\right) !}{\prod_{i \in[d]} r_{i} ! \prod_{i, j \in[d], k \in\left[n_{i}\right]} c_{i, j, k} !} \operatorname{det}\left(-k_{i j}\right) .
$$

From Proposition 5.3, we can also derive the number of the forests in $\mathcal{L}$ which was obtained in [5], see Proposition 2.

Proposition 5.4 (Enumeration of labeled plane forests by given numbers of different types of edges). Recall the definition of $k_{i j}^{\prime}, i, j \in[d]$ from Theorem 5.1. Then

$$
|\mathcal{L}|=\prod_{i, j=1}^{d}\left(n_{i}\right)^{k_{i j}^{\prime}} \frac{\prod_{j=1}^{d}\left(n_{j}-1\right) !}{\prod_{j=1}^{d} r_{j} ! \prod_{i, j \in[d]} k_{i j}^{\prime} !} \operatorname{det}\left(-k_{i j}\right) .
$$

Proof. A tuple $\mathbf{c}=\left(c_{i, j, k}\right)_{i, j \in[d], k \in\left[n_{i}\right]}$ of non-negative integers is an indegree tuple in $\mathcal{L}$ if and only if $\sum_{k=1}^{n_{i}} c_{i, j, k}=k_{i j}^{\prime}$ for $i, j \in[d]$. Define the set of indegree tuples $\mathbf{C}=\left\{\mathbf{c}=\left(c_{i, j, k}\right)_{i, j \in[d], k \in\left[n_{i}\right]} ; c_{i, j, k} \in \mathbb{Z}_{+}, \sum_{k=1}^{n_{i}} c_{i, j, k}=k_{i j}^{\prime}\right\}$. Summing all the indegree tuples $\mathbf{c} \in \mathbf{C}$, from Proposition 5.3, we obtain

$$
\begin{aligned}
|\mathcal{L}| & =\sum_{\mathbf{c} \in \mathbf{C}}|\mathcal{L}(\mathbf{c})|=\sum_{\mathbf{c} \in \mathbf{C}} \frac{\prod_{j=1}^{d}\left(n_{j}-1\right) !}{\prod_{j=1}^{d}\left(r_{j} ! \prod_{i \in[d], k \in\left[n_{i}\right]} c_{i, j, k} !\right)} \operatorname{det}\left(-k_{i j}\right) \\
& =\frac{\prod_{j=1}^{d}\left(n_{j}-1\right) !}{\prod_{j=1}^{d}\left(r_{j} ! \prod_{i \in[d]} k_{i j}^{\prime} !\right)} \operatorname{det}\left(-k_{i j}\right) \prod_{i, j=1}^{d}\left(\sum_{\left(c_{i, j,}, \dot{\mathbf{C}_{i j}}\right.} \frac{k_{i j}^{\prime} !}{\prod_{k=1}^{n_{i}} c_{i, j, k} !}\right) \\
& =\frac{\prod_{j=1}^{d}\left(n_{j}-1\right) !}{\prod_{j=1}^{d}\left(r_{j} ! \prod_{i \in[d]} k_{i j}^{\prime} !\right)} \operatorname{det}\left(-k_{i j}\right) \prod_{i, j=1}^{d}\left(n_{i}\right)^{k_{i j}^{\prime}}
\end{aligned}
$$


where $\mathbf{C}_{i j}=\left\{\left(c_{i, j, k}\right)_{k=1}^{n_{i}} ; c_{i, j, k} \in \mathbb{Z}_{+}, \sum_{k=1}^{n_{i}} c_{i, j, k}=k_{i j}^{\prime}\right\}$ for $i, j \in[d]$. (5.26) is obtained.

A multitype labeled plane forest is said to be injective if every vertex has at most one child of each type. Let $\mathcal{L}_{i n j}$ be the set consisting of injective forests in $\mathcal{L}$. Now we count the number of forests in $\mathcal{L}_{i n j}$. The following result was obtained in [3], see Proposition 9.

Proposition 5.5 (Enumeration of injective forests).

$$
\left|\mathcal{L}_{i n j}\right|=\prod_{i, j=1}^{d}\left(\begin{array}{c}
n_{i} \\
k_{i j}^{\prime}
\end{array}\right) \frac{\prod_{j=1}^{d}\left(n_{j}-1\right) ! \operatorname{det}\left(-k_{i j}\right)}{\prod_{j=1}^{d} r_{j} !} .
$$

Proof. If $\mathbf{c}=\left(c_{i, j, k}\right)_{i, j \in[d], k \in\left[n_{i}\right]}$ is the indegree tuple for an injective forest $\mathbf{f}$ in $\mathcal{L}_{i n j}$, then $c_{i, j, k}=0$ or 1 . Therefore, from Proposition 5.3,

$$
|\mathcal{L}(\mathbf{c})|=\frac{\prod_{j=1}^{d}\left(n_{j}-1\right) ! \operatorname{det}\left(-k_{i j}\right)}{\prod_{j=1}^{d}\left(r_{j} ! \prod_{i \in[d], k \in\left[n_{i}\right]} c_{i, j, k} !\right)}=\frac{\prod_{j=1}^{d}\left(n_{j}-1\right) ! \operatorname{det}\left(-k_{i j}\right)}{\prod_{j=1}^{d} r_{j} !} .
$$

This number is unrelated to the choice of indegree tuple c. Moreover, the forests in $\mathcal{L}_{i n j}$ have $\prod_{i=1}^{d} \prod_{j=1}^{d}\left(\begin{array}{c}n_{i} \\ k_{i j}^{\prime}\end{array}\right)$ different indegree tuples. Thus the cardinality of $\mathcal{L}_{i n j}$ is

$$
\left|\mathcal{L}_{i n j}\right|=\prod_{i, j \in[d]}\left(\begin{array}{c}
n_{i} \\
k_{i j}^{\prime}
\end{array}\right) \frac{\prod_{j=1}^{d}\left(n_{j}-1\right) ! \operatorname{det}\left(-k_{i j}\right)}{\prod_{j=1}^{d} r_{j} !} .
$$

Now we count the number of forests in $\mathcal{L}$ by fixing the number of vertices of each indegree type (but without fixing their labels). We say that a vertex has indegree type $\mathrm{u}=\left(u_{1}, u_{2}, \ldots, u_{d}\right)$ if it has $u_{j}$ children of type $j$ for $j \in[d]$. Let $\mathrm{U}=\prod_{i=1}^{d}\left\{0,1, \ldots, n_{i}\right\}$, and let $\mathrm{N}=\left(N_{i, \mathrm{u}}\right)_{i \in[d], \mathrm{u} \in \mathrm{U}}$ be a tuple of nonnegative integers satisfying $n_{i}=\sum_{\mathrm{u} \in \mathrm{U}} N_{i, \mathrm{u}}$ and $k_{i j}^{\prime}=\sum_{\mathrm{u} \in \mathrm{U}} u_{j} N_{i, \mathrm{u}}$ for $i, j \in[d]$. Denote by $\mathcal{L}(\mathrm{N})$ the subset of $\mathcal{L}$ in which the forests have $N_{i, \mathrm{u}}$ vertices of type $i$ with indegree type $\mathrm{u}$ for $i \in[d], \mathrm{u} \in \mathrm{U}$. Set $N(k)=$ $\sum_{i, j \in[d], u_{j}=k} N_{i, \mathrm{u}}+\sum_{i=1}^{d} \mathbf{1}_{\{k\}}\left(r_{i}\right), k=0,1, \ldots$ The following result is Proposition 12 in [3].

Proposition 5.6 (Enumeration of labeled plane forests by given the number of vertices of each indegree type). Given a tuple of nonnegative integers $\mathrm{N}=\left(N_{i, \mathrm{u}}\right)_{i \in[d], \mathrm{u} \in \mathrm{U}}$, satisfying the above assumptions, the number of forests in $\mathcal{L}(\mathrm{N})$ is

$$
|\mathcal{L}(\mathrm{N})|=\frac{\prod_{j=1}^{d}\left(n_{j}\right) !\left(n_{j}-1\right) !}{\prod_{i \in[d], \mathrm{u} \in \mathrm{U}}\left(N_{i, \mathrm{u}}\right) ! \prod_{k \geq 0}(k !)^{N(k)}} \operatorname{det}\left(-k_{i j}\right) .
$$

Proof. For any indegree tuple c such that $\mathcal{L}(\mathbf{c}) \subset \mathcal{L}(\mathrm{N}),(5.25)$ can be rewritten as

$$
|\mathcal{L}(\mathbf{c})|=\frac{\prod_{j=1}^{d}\left(n_{j}-1\right) !}{\prod_{k \geq 0}(k !)^{N(k)}} \operatorname{det}\left(-k_{i j}\right) .
$$

Since there are $\prod_{i \in[d]}\left(n_{i}\right) ! / \prod_{i \in[d], \mathrm{u} \in \mathrm{U}}\left(N_{i, \mathrm{u}}\right)$ ! different indegree tuples c for each given $\mathrm{N}$, the number of forests in $\mathcal{L}(\mathrm{N})$ is the product of the above two numbers, which gives (5.28).

We end this subsection with an enumeration of unlabeled forests. Define the subset $\mathcal{F}_{d}^{k_{i j}, \mathrm{n}}(\mathrm{N})$ of $\mathcal{F}_{d}^{k_{i j}, \mathrm{n}}$ consisting of unlabeled forests having $N_{i, \mathrm{u}}$ vertices of type $i$ with indegree type $\mathrm{u}$ for $i \in[d], \mathrm{u} \in \mathrm{U}$. 
Proposition 5.7 (Enumeration of plane forests by given the number of vertices of each indegree type).

$$
\left|\mathcal{F}_{d}^{k_{i j}, \mathrm{n}}(\mathrm{N})\right|=\frac{\prod_{j=1}^{d}\left(n_{j}-1\right) ! \operatorname{det}\left(-k_{i j}\right)}{\prod_{i \in[d], \mathrm{u} \in \mathrm{U}}\left(N_{i, \mathrm{u}}\right) !} .
$$

Proof. Recall that $N(k)=\sum_{i, j \in[d], u_{j}=k} N_{i, \mathrm{u}}+\sum_{i=1}^{d} \mathbf{1}_{\{k\}}\left(r_{i}\right), k=0,1, \ldots$ and define the canonical map $\Psi: \mathcal{L}(\mathrm{N}) \rightarrow \mathcal{F}_{d}^{k_{i j}, \mathrm{n}}(\mathrm{N})$, where for $\mathbf{f} \in \mathcal{L}(\mathrm{N}), \Psi(\mathbf{f})$ is the plane forest obtained by removing the labels of vertices in $\mathbf{f}$. Let $\mathbf{f}^{\prime} \in \mathcal{F}_{d}^{k_{i j}, \mathrm{n}}(\mathrm{N})$, and observe that for any $\mathbf{f}_{1}, \mathbf{f}_{2} \in \Psi^{-1}\left(\mathbf{f}^{\prime}\right)$ with respective indegree tuples $\mathbf{c}^{1}=\left(c_{i, j, k}^{1}\right)_{i, j \in[d], k \in\left[n_{i}\right]}$ and $\mathbf{c}^{2}=\left(c_{i, j, k}^{2}\right)_{i, j \in[d], k \in\left[n_{i}\right]}$, we have $\prod_{i, j \in[d], k \in\left[n_{i}\right]} c_{i, j, k}^{1} !=\prod_{i, j \in[d], k \in\left[n_{i}\right]} c_{i, j, k}^{2}$ ! Let us denote by $\prod_{i, j \in[d], k \in\left[n_{i}\right]} c_{i, j, k}$ ! this common value, then we readily check that

$$
\left|\Psi^{-1}\left(\mathbf{f}^{\prime}\right)\right|=\frac{\prod_{j=1}^{d} n_{j} !}{\prod_{i=1}^{d} r_{i} ! \prod_{i, j \in[d], k \in\left[n_{i}\right]} c_{i, j, k} !}=\frac{\prod_{j=1}^{d} n_{j} !}{\prod_{k \geq 0}(k !)^{N(k)}} .
$$

Then we derive from (5.28) that

$$
\left|\mathcal{F}_{d}^{k_{i j}, \mathrm{n}}(\mathrm{N})\right|=\frac{|\mathcal{L}(\mathrm{N})|}{\left|\Psi^{-1}\left(\mathbf{f}^{\prime}\right)\right|}=\frac{\prod_{j=1}^{d}\left(n_{j}-1\right) ! \operatorname{det}\left(-k_{i j}\right)}{\prod_{i \in[d], \mathrm{u} \in \mathrm{U}}\left(N_{i, \mathrm{u}}\right) !} .
$$

5.2. The Lagrange-Good inversion formula. Since the original paper by Good [10], the multivariate extension of Lagrange inversion formula has been widely studied by many authors. We refer to $[11,9,2,5]$ for different forms of Lagrange-Good inversion formula and proofs. The arborescent form of this result is introduced in [9] and [11], and is based on the notion of derivative with respect to a directed graph, see Definition 5.8 below. It is then is proved to be equivalent to the classical form. Here we will consider the arborescent form of this formula, as it fits properly to our setting. We will show that Theorem 1.2 implies the Lagrange-Good inversion formula. Although the latter is applicable for formal power series, here we only set up this formula for generating functions of probability distributions.

Definition 5.8 (Definition 1of [5]). Let $\mathcal{G}$ be a directed graph having $V=\{0,1,2,, \cdots, d\}$ as set of vertices and $E \subset V \times V$ as set of arcs(= directed edges), with the property that 0 has outdegree $d^{+}(0)$ equals to 0 . Let $\mathrm{g}(\mathrm{x})=\left(g_{0}(\mathrm{x}), g_{1}(\mathrm{x}), g_{2}(\mathrm{x}), \cdots, g_{d}(\mathrm{x})\right)$ be a vector of formal power series in $\mathrm{x}=\left(x_{1}, \cdots, x_{d}\right)$. We define the derivative of $\mathrm{g}(\mathrm{x})$ according to $\mathcal{G}$ by

$$
\frac{\partial \mathrm{g}(\mathrm{x})}{\partial \mathcal{G}}=\prod_{j \in V}\left\{\left(\prod_{(i, j) \in E} \frac{\partial}{\partial x_{i}}\right) g_{j}(\mathrm{x})\right\} .
$$

Recall that $D$ is the set of vectors $\left(j_{1}, j_{2}, \cdots, j_{d}\right), 0 \leq j_{i} \leq d$ such that $\left(j_{i}, i\right), i \in[d]$ codes an elementary forest as defined in Definition 4.3.

Definition 5.9. Denote by $g_{i}(\mathrm{x})$ a generating function of a probability distribution on $\mathbb{Z}_{+}^{d}, i=0,1, \cdots, d$. Set $\mathrm{g}(\mathrm{x})=\left(g_{0}(\mathrm{x}), g_{1}(\mathrm{x}), g_{2}(\mathrm{x}), \cdots, g_{d}(\mathrm{x})\right)$. Let $\mathrm{j}=\left(j_{1}, j_{2}, \cdots, j_{d}\right)$ be $a$ vector in $D$. Define the derivative of $\mathrm{g}(\mathrm{x})$ with respect to $\mathrm{j}$ by

$$
\frac{\partial \mathrm{g}(\mathrm{x})}{\partial \mathrm{j}}=\prod_{k=0}^{d}\left\{\left(\prod_{j_{i}=k} \frac{\partial}{\partial x_{i}}\right) g_{k}(\mathrm{x})\right\}
$$


where $\left(\prod_{j_{i}=k} \frac{\partial}{\partial x_{i}}\right)$ is equal to the identical operator when $\left\{i ; j_{i}=k\right\}=\emptyset$.

According to the definition of $D$, there exist a unique directed tree corresponding to any $\mathrm{j} \in D$. As a consequence and from Definitions 5.8 and 5.9, we see that the derivative of $\mathrm{g}(\mathrm{x})$ with respect to $\mathrm{j}$ is equal to the derivative of $\mathrm{g}(\mathrm{x})$ with respect to the corresponding tree of $\mathrm{j}$.

For example, for $d=2$, there are three elementary trees: $\mathrm{j}_{1}=(0,0), \mathrm{j}_{2}=(2,0)$, $\mathrm{j}_{3}=(0,1)$. The derivatives of the vector function $\mathrm{g}(\mathrm{x})=\left(g_{0}(\mathrm{x}), g_{1}(\mathrm{x}), g_{2}(\mathrm{x})\right)$ according to the vectors or the trees are

$$
\frac{\partial \mathrm{g}(\mathrm{x})}{\partial \mathrm{j}_{1}}=\frac{\partial^{2} g_{0}}{\partial x_{1} \partial x_{2}} \cdot g_{1} \cdot g_{2}, \quad \frac{\partial \mathrm{g}(\mathrm{x})}{\partial \mathrm{j}_{2}}=\frac{\partial g_{0}}{\partial x_{2}} \cdot g_{1} \cdot \frac{\partial g_{2}}{\partial x_{1}}, \quad \frac{\partial \mathrm{g}(\mathrm{x})}{\partial \mathrm{j}_{3}}=\frac{\partial g_{0}}{\partial x_{1}} \cdot \frac{\partial g_{1}}{\partial x_{2}} \cdot g_{2} .
$$

Let $f_{i}(\mathrm{x})$ denote the generating function of $\nu_{i}, i \in[d]$ and let $\nu_{0}$ be the Dirac measure on $\left(r_{1}, \cdots, r_{d}\right)$, that is $\nu_{0}=\delta_{\left(r_{1}, \cdots, r_{d}\right)}$. And set $f_{0}(\mathrm{x})=x_{1}^{r_{1}} \cdots x_{d}^{r_{d}},\left(x_{1}, \cdots, x_{d}\right) \in \mathbb{R}^{d}$, which is the generating function of $\nu_{0}$. For any $d$-dimensional nonnegative integer vector $\mathrm{m}=\left(m_{1}, m_{2}, \cdots, m_{d}\right)$, set $\mathrm{x}^{\mathrm{m}}=x_{1}^{m_{1}} \cdots x_{d}^{m_{d}}$. Then for any formal power series $h(\mathrm{x})$ with respect to $\mathrm{x}$, the coefficient of $\mathrm{x}^{\mathrm{m}}$ is denoted by $\left[\mathrm{x}^{\mathrm{m}}\right] h(\mathrm{x})$. In the remaining, without lose of generality, we assume that $d$-dimensional vector $\mathrm{n}$ is a positive integer valued vector. In our special setting, Arborescent Good-Lagrange formula can be stated as following.

Theorem 5.10. Let $f_{0}(\mathrm{x}), f_{1}(\mathrm{x}), \cdots, f_{d}(\mathrm{x})$ be given generating functions of offspring distributions and let $g_{i}(\mathrm{x}), i \in[d]$ be the generating functions of the total progeny distributions starting with one ancestor of type $i$. So that $g_{i}(\mathrm{x})=x_{i} f_{i}(\mathrm{~g})$ for $i \in[d]$, where $\mathrm{g}=\left(g_{1}, g_{2}, \cdots, g_{d}\right)$. Then

$$
\left[\mathrm{x}^{\mathrm{n}}\right] f_{0}(\mathrm{~g})=\left(\prod_{i=1}^{d} \frac{1}{n_{i}}\right)\left[\mathrm{x}^{\mathrm{n}-1_{d}}\right] \sum_{\mathrm{j} \in D} \frac{\partial\left(f_{0}(\mathrm{x}), f_{1}^{n_{1}}(\mathrm{x}), \ldots, f_{d}^{n_{d}}(\mathrm{x})\right)}{\partial \mathrm{j}},
$$

where $\mathrm{n}-1_{d}=\left(n_{1}-1, \ldots, n_{d}-1\right)$.

Proof. First note that the identity in Theorem 1.2 can be rewritten as

$$
\begin{aligned}
& \mathbb{P}_{\mathrm{r}}\left(O_{1}=n_{1}, \ldots, O_{d}=n_{d}, A_{i j}=k_{i j}, i, j \in[d], i \neq j\right) \\
= & \frac{\sum_{\left(j_{1}, \ldots, j_{d}\right) \in D} \prod_{i=1}^{d} k_{j_{i} i}}{n_{1} n_{2} \ldots n_{d}} \prod_{j=1}^{d} \nu_{j}^{* n_{j}}\left(k_{j 1}, \ldots, k_{j(j-1)}, n_{j}+k_{j j}, k_{j(j+1)}, \ldots, k_{j d}\right) \\
= & \left(\prod_{i=1}^{d} \frac{1}{n_{i}}\right)_{\left(j_{1}, \ldots, j_{d}\right) \in D}\left\{\left(\prod_{\left\{i ; j_{i}=0\right\}} r_{i}\right) \times\right. \\
& \left.\prod_{j=1}^{d}\left(\prod_{\left\{i ; j_{i}=j\right\}} k_{j i}\right) \nu_{j}^{* n_{j}}\left(k_{j 1}, \ldots, k_{j(j-1)}, n_{j}+k_{j j}, k_{j(j+1)}, \ldots, k_{j d}\right)\right\},
\end{aligned}
$$

where $\left(\prod_{\left\{i ; j_{i}=j\right\}} k_{j i}\right)=1$ when the product is taken over an empty set. Set the vectors $\mathrm{I}_{j}=\left(k_{j 1}, \ldots, k_{j(j-1)}, n_{j}+k_{j j}, k_{j(j+1)}, \ldots, k_{j d}\right), j \in[d], \mathrm{I}_{0}=\left(r_{1}, \ldots, r_{d}\right)$. Then $\prod_{\left\{i ; j_{i}=0\right\}} r_{i}=$ $\left[\mathrm{x}^{\mathrm{I}^{\prime}}\right]\left(\prod_{\left\{i ; j_{i}=0\right\}} \frac{\partial}{\partial x_{i}}\right) f_{0}(\mathrm{x})$, and for $j \in[d]$,

$$
\left(\prod_{\left\{i ; j_{i}=j\right\}} k_{j i}\right) \nu_{j}^{* n_{j}}\left(k_{j 1}, \ldots, k_{j(j-1)}, n_{j}+k_{j j}, k_{j(j+1)}, \ldots, k_{j d}\right)=\left[\mathrm{x}^{\mathrm{I}_{j}^{\prime}}\right]\left(\prod_{\left\{i ; j_{i}=j\right\}} \frac{\partial}{\partial x_{i}}\right) f_{j}^{n_{j}}(\mathrm{x})
$$


where $I_{j i}^{\prime}=I_{j i}-1$ when $i \in\left\{i ; j_{i}=j\right\}$, and $I_{j i}^{\prime}=I_{j i}$ when $i \notin\left\{i ; j_{i}=j\right\}, j=0,1, \ldots, d$. Since for $\left(j_{1}, \ldots, j_{d}\right) \in D$, each $i \in[d]$ appears exactly once in the sets $\left\{i ; j_{i}=j\right\}$, $j=0,1, \ldots, d, \sum_{j=0}^{d} \mathrm{I}_{j}^{\prime}=\mathrm{n}-1_{d}$. Note that $\left\{\mathrm{I}_{j} ; j \in[d]\right\}$ depend on $\left(k_{i j}\right)_{i, j \in[d]}$. Now fix n, $\mathrm{r}$ and $\mathrm{j}=\left(j_{1}, \ldots, j_{d}\right) \in D$. And define the set

$$
\mathrm{K}=\left\{\left(k_{i j}\right)_{i, j \in[d]} ; k_{i j} \in \mathbb{Z}_{+}, i \neq j,-k_{i i}=\sum_{j \neq i} k_{j i}+r_{i} \leq n_{i}\right\} .
$$

Take a sum for the term in the brace in (5.29) and express it in terms of characteristic functions:

$$
\begin{aligned}
& \sum_{\left(k_{i j}\right) \in \mathrm{K}}\left(\prod_{\left\{i ; j_{i}=0\right\}} r_{i}\right) \prod_{j=1}^{d}\left(\prod_{\left\{i ; j_{i}=j\right\}} k_{j i}\right) \nu_{j}^{* n_{j}}\left(k_{j 1}, \ldots, k_{j(j-1)}, n_{j}+k_{j j}, k_{j(j+1)}, \ldots, k_{j d}\right) \\
= & {\left[\mathrm{x}^{\mathrm{I}^{\prime}}\right]\left(\prod_{\left\{i ; j_{i}=0\right\}} \frac{\partial}{\partial x_{i}}\right) f_{0}(\mathrm{x}) \sum_{\left(k_{i j}\right) \in \mathrm{K}} \prod_{j=1}^{d}\left[\mathrm{x}^{\mathrm{I}_{j}^{\prime}}\right]\left(\prod_{\left\{i ; j_{i}=j\right\}} \frac{\partial}{\partial x_{i}}\right) f_{j}^{n_{j}}(\mathrm{x}) } \\
= & {\left[\mathrm{x}^{\mathrm{I}_{0}^{\prime}}\right]\left(\prod_{\left\{i ; j_{i}=0\right\}} \frac{\partial}{\partial x_{i}}\right) f_{0}(\mathrm{x}) \sum_{\sum_{j=0}^{d} \mathrm{I}_{j}^{\prime}=\mathrm{n}-1_{d}} \prod_{j=1}^{d}\left[\mathrm{x}^{\mathrm{I}_{j}^{\prime}}\right]\left(\prod_{\left\{i ; j_{i}=j\right\}} \frac{\partial}{\partial x_{i}}\right) f_{j}^{n_{j}}(\mathrm{x}) } \\
= & {\left[\mathrm{x}^{\mathrm{n}-1}{ }_{d}\right] \frac{\partial\left(f_{0}(\mathrm{x}), f_{1}^{n_{1}}(\mathrm{x}), \ldots, f_{d}^{n_{d}}(\mathrm{x})\right)}{\partial \mathrm{j}} . }
\end{aligned}
$$

Then we derive from this last computation that,

$$
\begin{aligned}
& {\left[\mathrm{x}^{\mathrm{n}}\right] f_{0}(\mathrm{~g})=\mathbb{P}_{\mathrm{r}}\left(O_{1}=n_{1}, \ldots, O_{d}=n_{d}\right) } \\
= & \sum_{\left(k_{i j}\right) \in \mathrm{K}} \mathbb{P}_{\mathrm{r}}\left(O_{1}=n_{1}, \ldots, O_{d}=n_{d}, A_{i j}=k_{i j}, i, j \in[d], i \neq j\right) \\
= & \sum_{\left(k_{i j}\right) \in \mathrm{K}} \sum_{\mathrm{j} \in D} \frac{\prod_{\left\{i ; j_{i}=0\right\}} r_{i}}{\prod_{i=1}^{d} n_{i}} \prod_{j=1}^{d}\left(\prod_{\left\{i ; j_{i}=j\right\}} k_{j i}\right) \nu_{j}^{* n_{j}}\left(k_{j 1}, \ldots, k_{j(j-1)}, n_{j}+k_{j j}, k_{j(j+1)}, \ldots, k_{j d}\right) \\
= & \left(\prod_{i=1}^{d} \frac{1}{n_{i}}\right) \sum_{\mathrm{j} \in D}\left[\mathrm{x}^{\mathrm{n}-1_{d}}\right] \frac{\partial\left(f_{0}(\mathrm{x}), f_{1}^{n_{1}}(\mathrm{x}), \ldots, f_{d}^{n_{d}}(\mathrm{x})\right)}{\partial \mathrm{j}} .
\end{aligned}
$$




\section{REFERENCES}

[1] K.B. Athreya And P. E. NeY: Branching processes. Die Grundlehren der mathematischen Wissenschaften, Band 196. Springer-Verlag, New York-Heidelberg, 1972.

[2] E.A. Bender, L.B. Richmond: A multivariate Lagrange inversion formula for asymptotic calculations. Electron. J. Combin., 5(1), (1998).

[3] O. Bernardi And A.H. Morales: Counting trees using symmetries. Preprint, arXiv:1206.0598, (2013).

[4] J. Bertoin: The structure of the allelic partition of the total population for Galton-Watson processes with neutral mutations. Ann. Probab. 37(4), 1502-1523, (2009).

[5] M. Bousquet, C.Chauve, G. Labelle,P. Leroux: Two bijective proofs for the arborescent form of the Good-Lagrange formula and some applications to colored rooted trees and cacti. Theoretical Computer Science 307, 277-302, (2003).

[6] A. CAYley: A theorem on trees. Quarterly Journal of Pure and Applied Mathematics, 23:376-378, 1889. (Also in The Collected Mathematical Papers of Arthur Cayley. Vol XIII, 26-28, Cambridge University Press, 1897).

[7] J.F. Le Gall: Random trees and applications. Probab. Surv., 2, 245-311, (2005).

[8] M. Dwass: The total progeny in a branching process and a related random walk. J. Appl. Probab. 6, 682-686, (1969).

[9] I.M. Gessel: A combinatorial proof of the multivariable Lagrange inversion formula J. Combin. Theory Ser. A 45(2), 178-195, (1987).

[10] I.J. Good: Generalizations to several variables of Lagrange's expansion, with applications to stochastic processes. Proc. Cambridge Philos. Soc. 56, 367-380, (1960).

[11] I.P. Goulden, D.M. Kulkarni: Multivariable Lagrange inversion, Gessel-Viennot cancellation, and the matrix tree theorem. J. Combin. Theory Ser. A80(2), 295-308, (1997).

[12] T.E. HARRIS: The theory of branching processes. Dover Phoenix Editions. Dover Publications, Inc., Mineola, NY, 2002.

[13] T.E. Harris: First passage and recurrence distributions. Trans. Amer. Math. Soc., 73, 471-486, (1952).

[14] I. KoRTCHemsKi: Invariance principles for Galton-Watson trees conditioned on the number of leaves, Stoch. Proc. Appl., 122, 3126-3172, (2012).

[15] G. Miermont: Invariance principles for spatial multitype Galton-Watson trees. Ann. Inst. Henri Poincaré Probab. Stat., 44, no. 6, 1128-1161, (2008).

[16] N. Minami: On the number of vertices with a given degree in a Galton-Watson tree. Adv. Appl. Probab 37, 229-264 (2005).

[17] C.J. ModE: Multitype branching processes. Theory and applications. Modern Analytic and Computational Methods in Science and Mathematics, No. 34 American Elsevier Publishing Co., Inc., New York 1971.

[18] J.W. Moon: Some determinant expansions and the matrix-tree theorem. Discrete Math. 124, no. $1-3,163-171,(1994)$.

[19] R. OtTER: The multiplicative process. Ann. Math. Statistics 20, 206-224, (1949).

[20] J. Pitman: Combinatorial Stochastic Processes. Saint-Flour, Springer, 2002.

[21] J. Pitman: Enumerations of trees and forests related to branching processes and random walks. Microsurveys in discrete probability (Princeton, NJ, 1997), 163-180, DIMACS Ser. Discrete Math. Theoret. Comput. Sci., 41, Amer. Math. Soc., Providence, RI, 1998.

[22] R.P. Stanley: Enumerative combinatorics. Volume 1. Second edition. Cambridge Studies in Advanced Mathematics, 49. Cambridge University Press, Cambridge, 2012.

[23] W.T. Tutte: The dissection of equilateral triangles into equilateral triangles. Proc. Cambridge Philos. Soc. 44, 463-482, (1948).

LoḮ Chaumont - LAREMA - UMR CNRS 6093, Université D'Angers, 2 Bd Lavoisier, 49045 Angers CEDEX 01

E-mail address: loic.chaumont@univ-angers.fr

Rongli Liu - Department of Mathematics, Nanjing University, Nanjing, 210093, P.R.China E-mail address: rongli.liu@gmail.com 